Mediterránea Ser. Biol. (1991), n. 13. Pág. 27-66

\title{
I SERPENTI DELL'EMILIA-ROMAGNA E IN PARTICOLARE DELLA PROVINCIA DI BOLOGNA (ITALIA SETTENTRIONALE)
}

\author{
por \\ ETTORE BASSINI ${ }^{1}$, SILVIO BRUNO ${ }^{2}$, PAOLO MAZZEI ${ }^{3}$, \\ GUGLIELMO STAGNI ${ }^{4}$
}

\section{SUMMARY}

Geographical distribution and some morphological aspects of Emilia-Romagna snakes, and particularly of the Bologna Province (North Italy), are discussed.

The study is based on about 6.000 reptiles, 4.185 of which are Vipera aspis, collected or killed during 7 years in the Bologna Province.

Coronella girondica, Elaphe Iongissima and Vipera aspis seems to be absent today in the vegatational lower lever. Coluber viridiflavus, Natrix natrix and Natrix tessellata seems to be more spread on the hills and on the mountanis than on the plain. Coronella austriaca was found only on the plain.

Elaphe quatuorlineata was recorded in many North Italy localities. Probably sometimes it was mistaken with the back lined Elaphe longissima and sometimes the recorded specimens were introduced (normally without acclimatization from Central Italy or West Jugoslavija. On the other hand it is possible that during XIX century a relicted population was present in the Bologna surroundings.

The recent recordings for the Romagna Region are doubtful and those for the Piacenza Province are probably referred to indigenous relict specimens or to imported and acclimatizated ones.

In Italy Elaphe situla is present surely in Sicilia (especially in the East), in the East Basilicata and in the South and Central Puglia.

It was mentioned for the SE Basilicata in many news of the XIX Century end and in papers of ' 50 and ' 60 of the XX Century, but its current presence has to be confirmed.

The specimens recorded in Emilia Romagna were imported or were mistaken with Coronella girondica or with Elaphe quatuorlineata youngs (perhaps alloctonous). In the XIX Century second half it was mentioned for the Abruzzo (Central Italy), but probably a locality mistake exists: Abruzzo instead Dalmatia.

\footnotetext{
${ }^{1}$ Riccrcatore dell'Istituto Nazionale di Biologia della Selvaggina (oggi Istituto Nazionale per la Fauna Selvatica), via Cà Fornacetta 9, 40064 Ozzano dell’Emilia (Bologna).

2 Via di Pizzo Morronto 43, 00061 Anguillara Sabazia (Roma).

${ }^{3}$ Via Passo del Furlo 19, 00141 Roma.

${ }^{4}$ Via Molino Mingano 1, Roncastaldo (40050 Loiano, BO).
} 
Contrary to what believed until ' 70 of this Century, Natrix maura in Emilia Romagna is apparently more localized than rare and, perhaps, more spread than deducible from the bibliography.

During XIX Century and the beginning of XX one, Vipera berus was present in many localities of the Po Plain. After about 1915-1918 it was not observed again. Probably it came extincted because of different anthropic reasons. In Italy is actually present in the Alpine Region. Anyway times to times, especially after years ' 50 , it was recorded in Emilia Romagna at the Apennine Mounts base. During July 1969 a Joung specimen was collected in the Sasso Marconi territory (Bologna Pre-Appennine) and during summer 1983 another joung was captured at the Salse di Nirano (Modena). We don't know if those specimens are autochthonous.

More researches to confirm the presence of this species in Emilia Romagna have to be carried on.

\section{PREMESSA}

In teoria, le specie di serpenti che vivono in Emilia-Romagna dovrebbero essere conosciute. In realtà, la presenza attuale del marasso (Vipera berus) è messa in discussione o esclusa, il cervone (Elaphc quatuorlineata) è incluso tra gli ofidi indigeni da alcuni ricercatori, ma rifiutato da altri che lo considerano importato e non acclimatato, e la ripartizione della biscia viperina (Natrix maura) è in gran parte ignota. Inoltre le conoscenze sulla distribuzione - spaziale e altitudinale- dei serpenti nella regione sono più potenziali e reali perché basate, almeno in alcuni casi, su notizie storiche o di 20-30 anni fa.

Per rimediare a queste lacune, l'Istituto per i Beni Artistici, Culturali e Naturali della Regione Emilia-Romagna (IBACN) ha iniziato, dal 1981, uno studio dell'erpetofauna regionale svolto "sul campo" da G. Stagni (per i rettili) e da S. Mazzotti (per gli anfibi).

Nella prima metà degli anni Settanta, il prof. A. Toschi —allora direttore del Laboratorio di Zoologia applicata alla Caccia (LZAC), l'attuale Istituto Nazionale per la Fauna Selvatica (INFS) - diede a due di noi (E. Bassini e S. Bruno) l'incarico di esaminare i circa 6.000 rettili (perlopiú serpenti) avuti dall'Aministrazione Provinciale di Bologna (APB) e catturati nella provincia omonima durante la "Campagna Antivipera" promossa da questa amministrazione negli anni 1966, 1967, 1968, 1969, 1970, 1971 e 1973'.

Ogni esemplare di rettile, ucciso e consegnato, era accompagnato da una apposita scheda dell'APB (fig. 1) e la determinazione specifica degli animali -anche a causa del premio in lire erogato dall'APB se si trattava di viperidi- era controllata dai ricercatori del LZAC di Bologna.

In 7 anni furono portati al LZAC diverse migliaia di serpenti, provenienti da tutto il Bolognese, la maggior parte dei quali —per l'esattezza 4.185 individui- erano vipere comuni (Vipera aspis).

Purtroppo, la maggioranza dei serpenti consegnati erano in condizioni fisiche disastrose, perché avevano il corpo a pezzi o erano rappresentati solo dalla testa. Di conseguenza tutti questi esemplari, disastrati e inservibili, furono eliminati. Invece, quelli in buono stato (oltre 1.000 ) vennero conservati, catalogati e registrati su apposite schede (fig. 2). Quest'ultima fase dell'operazione fu poi largamente svolta da G. Stagni, che studiò gran parte degli ofidi preservati per la sua tesi di laurea in scienze naturali.

Le osservazioni che seguono scaturiscono dalle ricerche dell'IBACN, dal materiale ofidiologico consegnato all'INFS (già LZAC), e oggi solo in parte qui custodito, e dalle indagini naturalistiche, promosse dall'Ente Nazionale per l'Energia Nucleare e per l'Energie Alternative (ENEA), nell'Appennino Bolognese nell'autunno 1987 e nell'estate 1989. 
AMMinistrazione PROVINCIALE D1 BOLOGNA

CAMPAgBa AvTHVIRERA

$12-5-5 t$

№ 0108

\section{Catturato da:}

Nome o Gogame Stoke

Indizizo S.Morea Ocicua Pinuaro

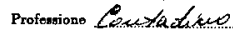

Localit Tragela

deta cattura $10-5-57$

II rettle viene conscgnato dis

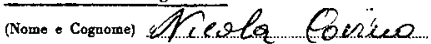

Indirizzo

IL MCEVENTE
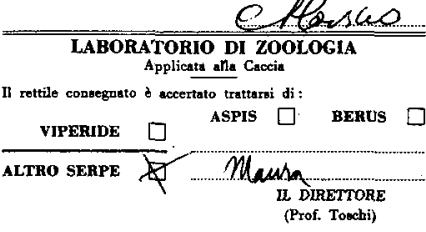

Figura 1.- Facsimile di una delle schede fatte stampare dall'Amministrazione Provinciale di Bologna per la "Campagna Antivipera" promossa dal 1966 al 1973 (1972 escluso).

Tutti i serpenti raccolti circa 6.000 (nel totale bisogna però considerare anche più sauri: luscengole, orbettini, ecc.) - dovevano essere consegnati al Laboratorio di Zoologia applicata alla Caccia per un'attendibile identificazione. Il premio in lire veniva corrisposto soltanto a chi uccideva una o più vipere e poteva esibirne i resti (testa, corpo o esemplare integro). Nella figura è riprodotta la scheda compilata di una supposta vipera comune: in realtà una biscia viperina.

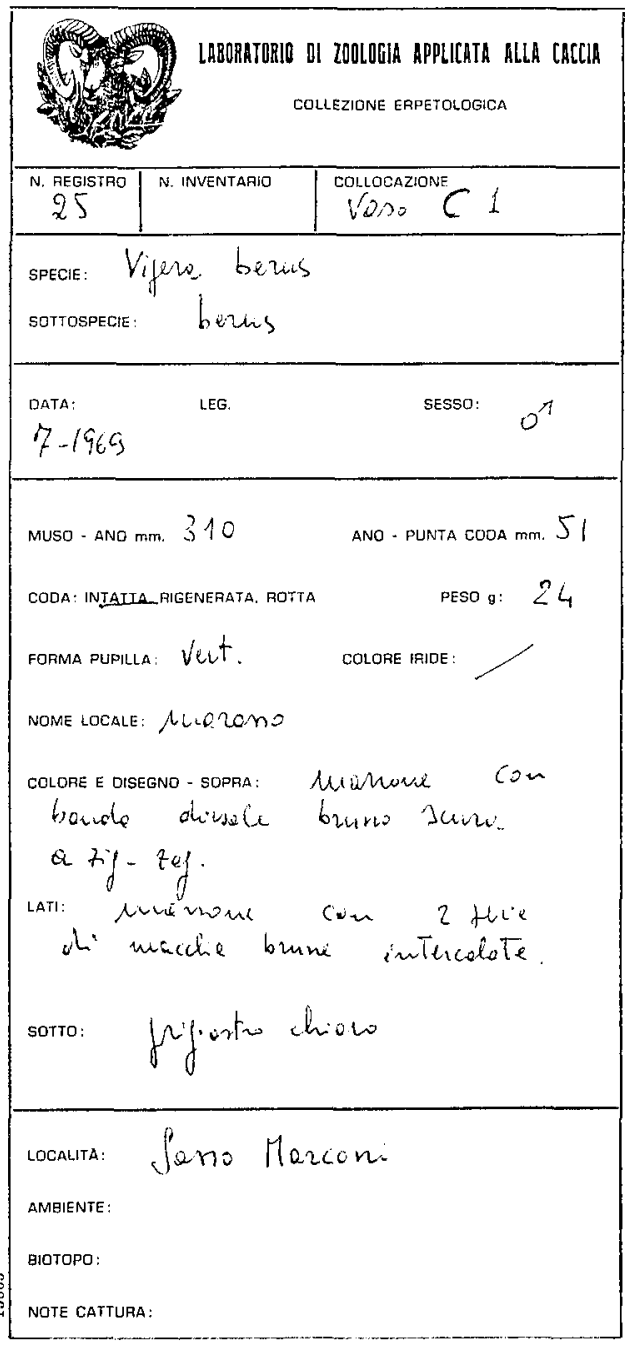

Figura 2.- Tutti i rettili in buone condizioni, raccolti nel Bolognese durante la "Campagna Antivipera" e consegnati al Laboratorio di Zoologia applicata alla Caccia, furono incorporati nella Collezione Erpetologica di questo Istituto e dotati di una scheda individuale. Qui reproduciamo la scheda del marasso. 


\section{INTRODUZIONE}

Il Bolognese è una delle 8 province di cui è composta amministrativamente la regione italiana Emilia-Romagna. La superficie dei 60 territori comunali (fig. 6) che costituiscono la provincia di Bologna è di $3.702 \mathrm{~km}^{2}$.

Il clima e la vegetazione del Bolognese - i due aspetti dell'ambiente fisico della provincia che più ci interessano per il suo popolamento ofidico- li abbiamo sintetizzati nella fig. 3, tratta da G.C. Carloni, F. Francavilla e F. Corbetta (1974).

Nel testo facciamo abitualmente uso di terminologie bioclimatiche, biogeografiche e vegetazionali, i cui concetti sono stati chiariti e illustrati, per esempio, da Saint Girons (1952a, 1982a,b) Lattin (1967), Tomaselli et alii (1973), Pignatti (1979), Ferrari et alii (1980), Müller (1981) e Bertolani Marchetti (1982).

Abbiamo considerato tutte le 11 specie di serpenti segnalati in letteratura dell'Emilia-Romagna; 9 di esse risultano presenti in provincia di Bologna. Nell'ambito degli ofidi stimati, 2 appartengono alla famiglia dei viperidi (Vipera berus e Vipera aspis) e 9 a quella dei colubridi (Natrix tessellata, Natrix natrix, Natrix maura, Elaphe situla, Elaphe quatuorlineata, Elaphe longissima, Coronella girondica, Coronella austriaca e Coluber viridiflavus).

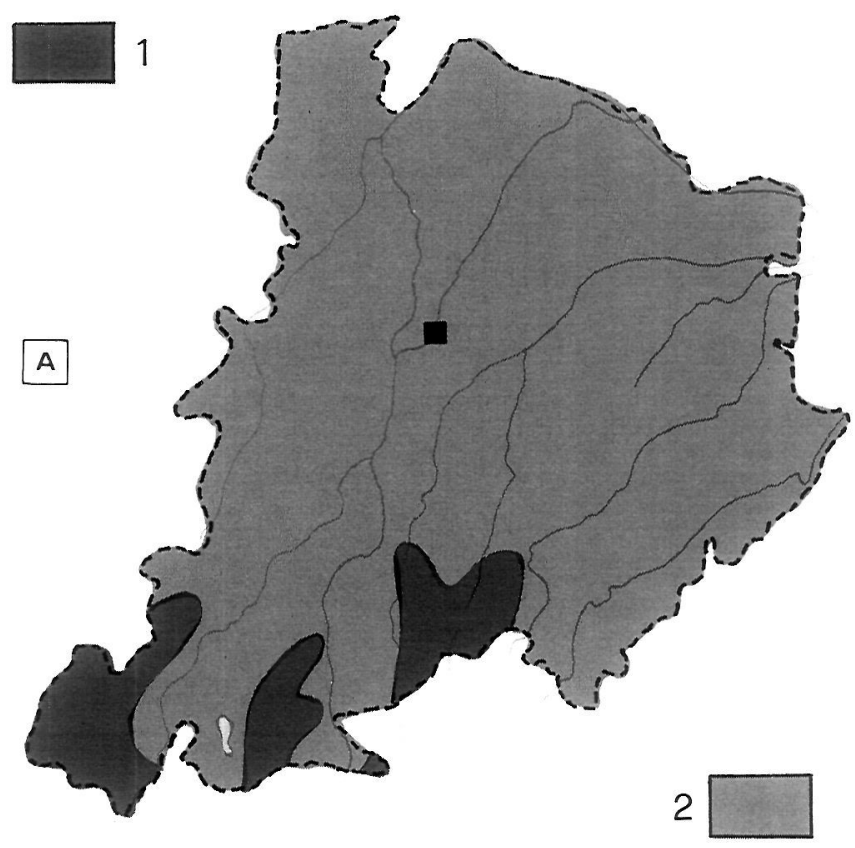

Figura 3 A.- Aspetti bioclimatici della provincia di Bologna. Temperature medie di gennaio (A): $-0^{\circ} \mathrm{C}(1)$, da $0^{\circ}$ a $4{ }^{\circ} \mathrm{C}(2)$. 


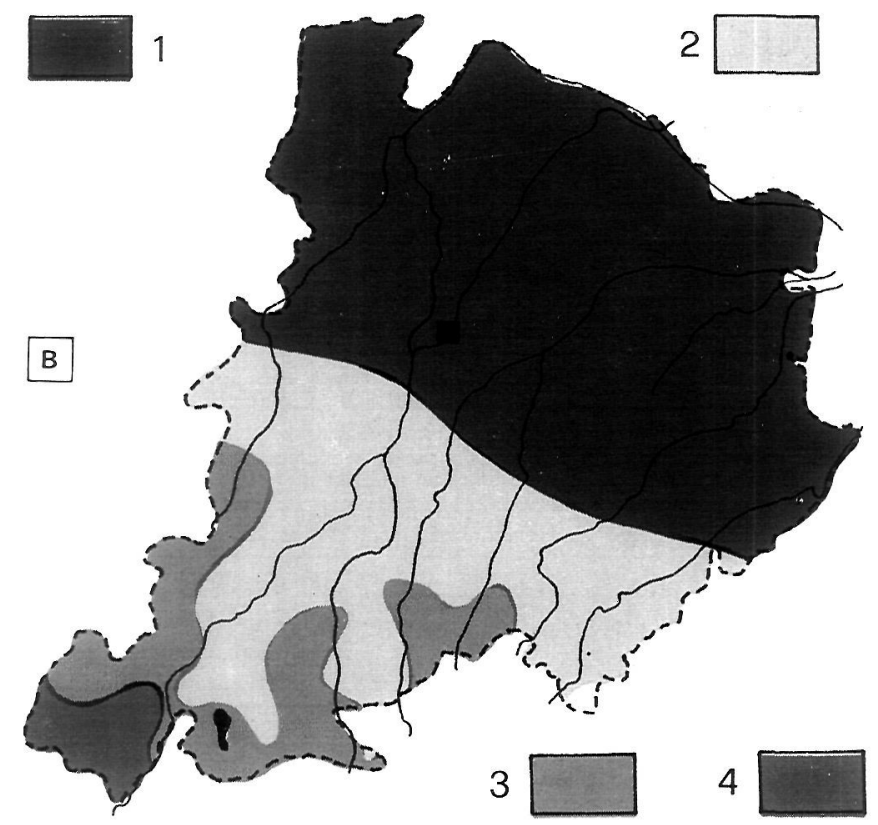

Figura 3 B.- Aspetti bioclimatici della provincia di Bologna. Temperature medie di luglio (B): oltre $24^{\circ} \mathrm{C}(1)$, da $20^{\circ}$ a $24^{\circ} \mathrm{C}(2)$, da $16^{\circ}$ a $20^{\circ} \mathrm{C}$ (3), meno di $16^{\circ} \mathrm{C}$ (4).

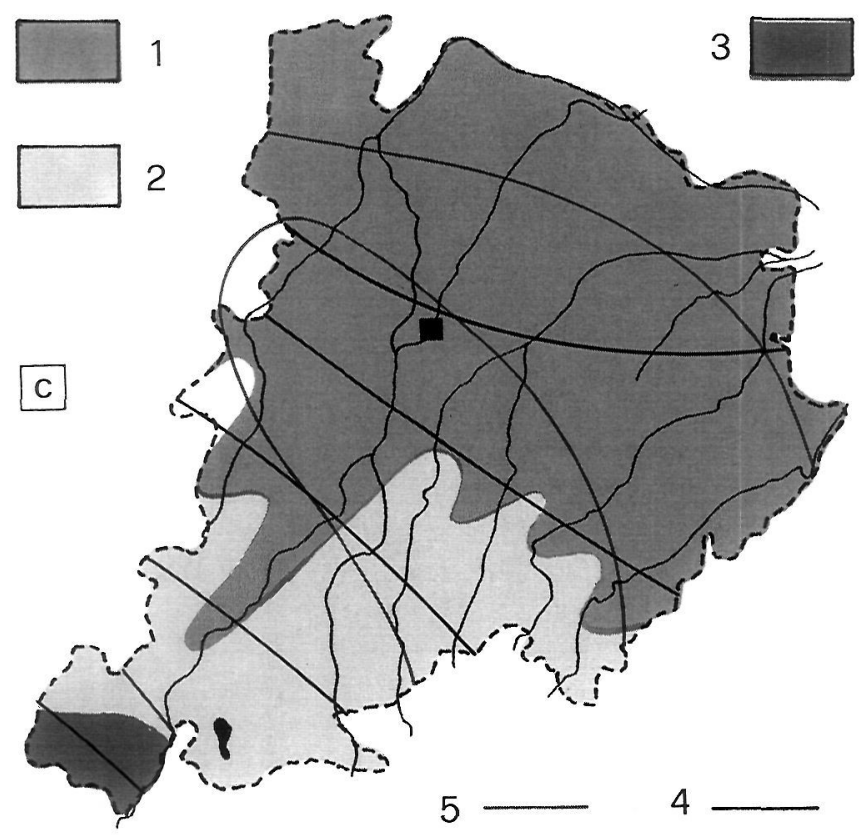

Figura 3 C.- Aspetti bioclimatici della provincia di Bologna. Precipitazione medie annue (C): meno di $1.000 \mathrm{~mm}$ (1), da 1.000 a $1.500 \mathrm{~mm}$ (2), da 1.500 a $2.000 \mathrm{~mm}$ (3), isoterme di gennaio (4), isoterme di luglio (5). 


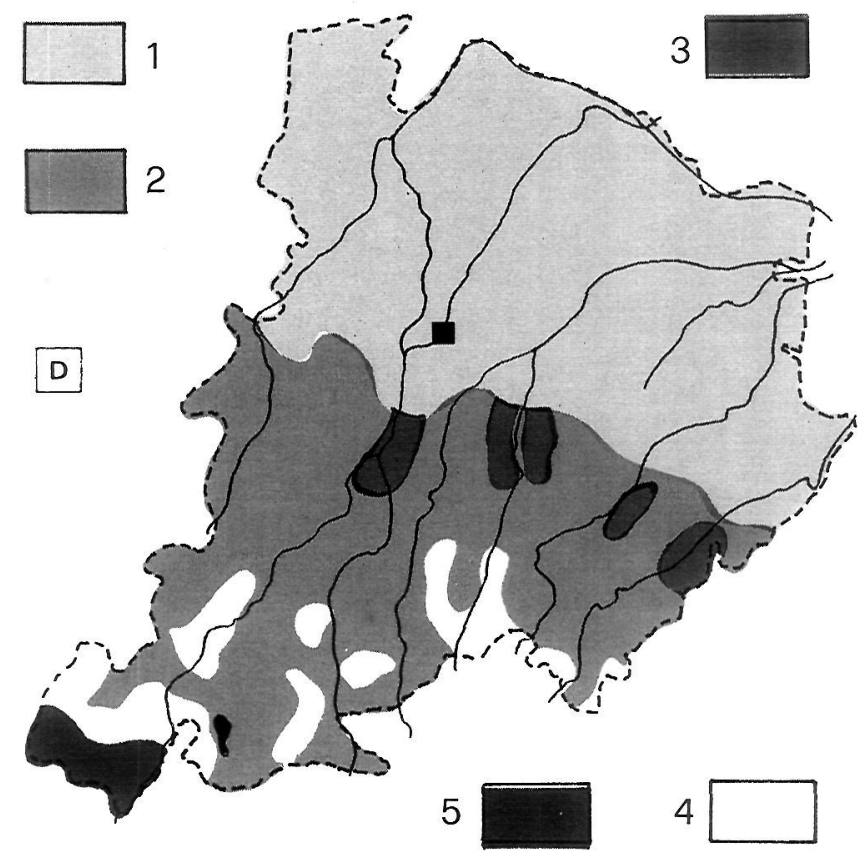

Figura 3 D.- Aspetti bioclimatici della provincia di Bologna. Formazioni vegetazionali (D): fascia della farnia e del carpino (con Quercus robur, Ostrya carpinifolia, Fraxinus oxycarpa, Salix alba, Alnus glutinosa, Populus alba, Ulmus campestre, ecc) (1), fascia della roverella (Quercus pubescens) e del cerro (Quercus cerris) (2), fascia del leccio (Quercus ilex) (3), fascia del faggio (Fagus sylvatica)(4), fascia della brughiera culminale o di vetta (5).

La letteratura ofidiologica dell' Emilia- Romagna è più ampia di quella riportata nella nostra bibliografia. In questa sede abbiamo esaminato, soprattutto, oltre alle informazioni — storiche e attuali, edite e parzialmente inedite- sulla provincia di Bologna (cfr. per esempio Bianconi 1867, Bruno e Maugeri 1977, Ciucci e Fumagalli 1981, Cocconi 1881, Günther 1858, Lorenzini 1914, Naviglio et alii 1989, Zaffagnini e Bigazzi 1975) anche quelle su altre provincie regionali (cfr. per es. Aa.Vv. 1986, Bernardino et alii 1977, Bertusi 1982, Boldreghini 1983, 1987, Bonizzi 1870, 1881, Bruno e Maugeri 1977, Carruccio 1882a,b, de Marchi 1974, 1980, Del Prato 1887, 1899, Imparati 1940, Jan 1863, Niekisch 1982, Picaglia 1885, Piccoli 1983, Rabacchi 1984, Rossi e Mezzadri 1983, Rubbi e Clò 1980, Senni 1985, Silvestri 1972, 1978, Toschi 1963, 1974, Vandoni 1914, Zangheri 1961, 1969) allo scopo di presentare un quadro moderno e ragionato sulla distribuzione e la variabilità morfologico-geografica dei serpenti considerati.

Per ogni ulteriore referenza storica rimandiamo al "Repertorio" di Bruno (1992). 


\section{SPECIE OFIDICHE}

\section{Marasso [Vipera berus (Linnaeus, 1758)]}

La specie è un'elemento faunistico arboreale manciuriano-sibirico, a corologia eurocentrosettentrionale-centroasiatica. In Italia il marasso era considerato, almeno fino al 1889 , un rettile caratteristico dell'ambiente collinoplaniziare della fascia di vegetazione medioeuropea; dal 1915-1918 circa ad oggi è, invece, giudicato un rettile tipico degli ambienti subalpino e alpino delle fasce di vegetazione boreale e alpica (fig. 4).

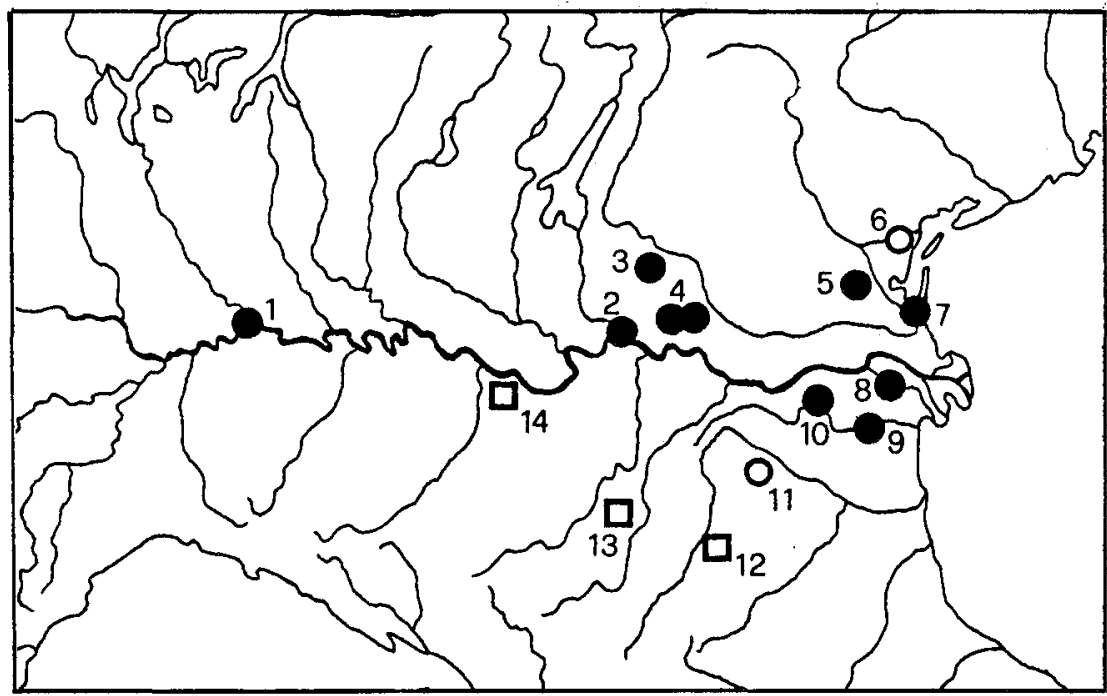

Figura 4.- Distribuzione approssimativa e semplificata, storica e (?) attuale, del marasso ( $V i$ pera berus) nella Padania e dintorni.

Cerchi neri: località storiche dove l'ofidio era certamente presente, forse ancora agli inizi del 1900 , e dove la maggior parte degli studiosi ritengono che sia probabilmente scomparso per cause antropiche. Cerchi bianchi: località storiche dove il rettile era forse presente. Quadrati: località attuali (12 e 13 reali, 14 ipotetica) dove la presenza del marasso deve essere confermata $(12,13)$ e dimostrata (14).

Provincia di Pavia (Lombardia): lungo le risaie e le paludi del Po e del Ticino, 65-85 m (1). Provincia di Mantova (Lombardia): tra Gazzo, Governolo, Ostiglia, Sustinente e Villimpenta, cioè tra i corsi dei fiumi Po e Tione, $10-20 \mathrm{~m}(2)$. Provincia di Verona (Veneto): dintorni di Zevio, $30 \mathrm{~m}$ circa (3). Provincia di Verona (Veneto): dintorni di Casaleone, Legnago e Roncanova, cioè tra i corsi dei flumi Adige, Tartaro e Tione (Grandi Valli Veronesi), 15-20 m (4). Provincia di Padova (Veneto): dintorni di Piove di Sacco, $5 \mathrm{~m}$ circa (5). Provincia di Venezia (Veneto): territorio lagunare e dintorni, 0-5 m (6). Provincia di Venezia (Veneto): dintorni del Forte di Chioggia, $2 \mathrm{~m}$ circa (7). Provincia di Rovigo (Veneto): Isola d'Ariano (Polesine), 0-2 m (8). Provincia di Ferrara (Emilia-Romagna): dintorni di Codigoro, 2-5 m (9). Provincia di Ferrara (Emilia-Romagna): dintorni di Copparo, $1-5 \mathrm{~m}$ (10). Provincia di Bologna (Emilia-Romagna): dintorni di Malalbergo, $10 \mathrm{~m}$ (11). Provincia di Bologna (Emilia-Romagna): dintorni di Sasso Marconi, il centro abitato sorge a $124 \mathrm{~m}$ d'altitudine (12). Provincia di Modena (Emilia-Romagna): Salse di Nirano (Fiorano Modenese), $195-220 \mathrm{~m}$ circa (13). Provincia di Parma (Emilia-Romagna): lungo il corso del Po, 25-29 m (14). 
La presenza di Vipera berus in Emilia-Romagna era documentata per la provincia di Ferrara (Jan 1863, Boulenger 1896): Codigoro "dove la specie è molto comune" (De Betta 1879, 1880) e Copparo (Camerano 1889) ${ }^{2}$. Nel 1914, tuttavia, Vandoni non segnala il marasso per il Ferrarese, forse perchè riteneva che qui fosse estinto. Nel 1975, nondimeno, Zaffagnini e Bigazzi scrivono che in Emilia-Romagna il Viperidae vive "solo nel ferrarese, dove però è molto raro". Noi non abbiamo informazioni recenti o dirette al riguardo.

Recentemente de Marchi $(1974,1980)$ ha segnalato il marasso per la provincia di Parma dove "sempre raro, sembra sia divenuto alquanto più frequente in questi ultimi anni. (...) Si può incontrare lungo le rive del Po". Anche per questa zona manchiamo di osservazioni personali.

Già Aldrovandi e Ambrosino (1639) affermavano che l'attuale Vipera berus fosse comune nel Bolognese, in particolare presso Malalbergo. Purtroppo, sia questa notizia che quelle di altri studiosi ${ }^{3}$, relative alla presenza del marasso nella pianura Bolognese, sembrano poco documentate. Anche le attestazioni del 1974, 1975 e 1980 devono, in mancanza di prove incontestabile in merito, accogliersi con riserva.

Toschi (1974) ricorda la specie per l'Appennino Emiliano e a S. Bruno, che a voce gli chiedeva maggiori informazioni in merito, rispose che la presenza del marasso era attestata da più naturalisti e cacciatori emiliani che lui giudicava degni di fede.

Nell'ambito degli ofidi raccolti durante la "Campagna Antivipera", promossa dall'APB e portati al INFS (LZAC), c'è un maschio di Vipera berus (collezione erpetologica n. 25, vaso C1) lungo $361 \mathrm{~mm}$ (di cui $51 \mathrm{~mm}$ spettanti alla coda) catturato nel luglio 1969 a Sasso Marconi.

Questo reperto è attendibile? A parte la lacuna del nome della località (nelle schede di cattura, infatti, è quasi sempre riportato il nome del Comune o della Frazione dove risiede il catturatore, invece dell'esatta località di provenienza dell'esemplare), si potrebbe anche supporre che il marasso sia stato raccolto altrove - per esempio in Trentino, dove l'ignoto raccoglitore emiliano era magari andato in vacanza o acaccia - e poi consegnato all'APB per riscuotere i soldi della taglia.

Nel 1984, tuttavia, i naturalisti fioranesi Callegari, Ballei, Termanini e Parisi pubblicavano la foto a colori di una Vipera berus catturata nel Parco Naturale Regionale delle Salse di Nirano (Modena).

Nello stesso anno Rabacchi scriveva che "eventuali segnalazioni o catture [nel Modenese] del Marasso, Vipera berus, debbono essere (...) attentamente vagliate. Nella maggioranza dei casi si è infatti appurato trattarsi di esemplari di Aspide con colorazioni non del tutto tipiche ${ }^{4}$. Non si può escludere tuttavia la presenza di qualche Marasso proveniente da altre regioni dell'Italia settentrionale giunto sino a noi nascosto tra carichi di legname o di fieno".

L'individuo in questione - un maschio lungo circa $38 \mathrm{~cm}$ e di cui 4,3 $\mathrm{cm}$ spettano alla coda, con 146 piastre ventrali e 37 paia di piastre sottocodali- è oggi conservato nel Museo Naturalistico del Gruppo Ecologico Fio- 
ranese a Fiorano Modenese. L'esemplare fu catturato nell'estate del 1983 $\mathrm{e}$ tenuto in vita più giorni dai coniugi $\mathrm{A}$. Bellei e $\mathrm{L}$. Callegari per la loro attività didattico-naturalistica presso gli istituti scolastici del comprensorio fioranese (tav. 1, fig. 1).

Due brevi sopraluoghi alle Salse di Nirano, svolti da S. Bruno, L. Callegarri e P. Mazzei nella seconda metà del pomeriggio del 18 settembre 1987 e nella prima metà della mattina del giorno dopo, hanno rilevato la presenza dei rettili Coluber viridiflavus, Natrix natrix, Lacerta viridis, Podarcis muralis (sia a dorso bruno che a dorso verde) e Podarcis sicula. Contrariamente a quanto si verifica di solito a settembre -in un'area collinoplaniziare a clima temperato che rientra nel climax del querceto a roverella (Quercus pubescens) - la temperatura e l'umidità dell'aria furono decisamente sfavorevoli alla fuoriuscita o comunque all'attività (anche di tipo secondario o ridotto) di un Viperidae a selezione termica, come è appunto Vipera berus. Il tipo di vegetazione e l'ambiente naturale, però, sono simili a quelli che si trovano, per esempio, in alcune zone, del Sussex e del Kent (Inghilterra) o della Scandinavia, frequentate dal serpente.

Ferrari et alii (1980), a proposito delle Salse di Nirano, scrivono che qui "è stata descritta una vegetazione dominata da Agropyron litorale, Puccinellia distans e Bupleurum tenuissimum, per alcuni aspetti simile a quella delle steppe salate centroasiatiche e agli Agropireti del litorale nordatlantico. La presenza di Puccinellia distans, una graminacea, è di particolare interesse: questa specie alofila, suddivisa in numerose sottospecie, ha una distribuzione prevalentemente litorale in Europa e in Siberia e qui si trova invece in un'area decisamente <continentale〉».

Date queste premesse (cfr. al riguardo anche gli scritti di Bertolani Marchetti del 1980, 1982 e 1983), Vipera berus, forse estinta nella Pianura Padana, potrebbe essere sopravissuta in alcune salse ${ }^{5}$ dell'Appennino Emiliano e in certe località del Preappenino.

Ricerche in merito sono indispensabili, anche al fine di verificare i due singolari reperti qui avanzati ${ }^{6}$.

Secondo alcuni studiosi i marassi della Padania appartengono alla sottospecie Vipera berus marasso (Pollini, 1818), che la letteratura tossonomica classica considera sinonimo della sottospecie tipo o tipica o nominale e cioè di Vipera berus berus.

\section{Vipera comune [Vipera aspis (Linnaeus, 1758)]}

Abbiamo avuto la possibilità di esaminare 4.185 esemplari di questa specie catturati in provincia di Bologna tra il 1966 e il 1973. Gli individui rovinati o letteralmente massacrati sono stati eliminati perché inservibili. Nell'ambito di quelli ritenuti meritevoli di essere conservati, circa 500, tra adulti e subadulti di entrambi i sessi, sono stati analizzati morfologicamente da G. Stagni del cui studio, oggetto di tesi (1984) e di prossima pubblicazione, anticipiamo alcuni risultati.

Nel Bolognese, la vipera comune è distribuita esclusivamente sulle colline e sulle montagne a sud della Via Emilia. La frequenza della specie è 
maggiore oltre i $500 \mathrm{~m}$ di quota e, più in generale, aumenta con l'altitudine (fino a $1.560 \mathrm{~m}$ ) probabilmente per la parallela diminuzione di effetti antropici negativi e per la conseguente maggiore disponibilità a varietà di ambienti. Inoltre la densità del rettile sembra essere più elevata nella parte occidentale della provincia (a ovest del fiume Reno) (fig. 5).

I maschi raggiungono lunghezze totali maggiori di quelle delle femmine, sia in assoluto che in media. Tali differenze ( $\mathrm{e}$ in particolare quella dei valori medi: $60,69 \mathrm{~cm}$ in 291 maschi e $58,22 \mathrm{~cm}$ in 209 femmine) potrebbero, comunque, dipendere da motivi connessi con la particolare biologia della specie, piuttosto che da effettive diversità morfologiche delle popolazioni.

Confermiamo, come caratteri nettamente legati il sesso, ek numero delle piastre sottocodali o urostegi, quello delle macchie scure che ornano la parte superiore della coda e alcuni aspetti della livrea. Inoltre, contrariamente a quanto è finora noto, si rivelano significativamente dipendenti dal sesso anche il numero delle piastre ventrali o gastrostegi e quello delle macchie scure trasversali che ornano il dorso.

I campioni di collina e di montagna presentano disomogeneità significative riguardo al grado di frammentazione delle placche cefaliche o sincipitali (più elevato in montagna) e all'ornamentazione dorsale (più monotona in collina) e rivelano l'esistenza di influenze altitudinali-ambientali la cui portata e il cui significato andrebbero approfonditi da ulteriori ricerche.

Vipera aspis, come risulta dalla sua ampia letteratura (cfr. per esempio Naulleau 1966a,b, Saint Girons 1952b), è specie sedentaria e di solito poco incline a frequentare ambienti umidi. Pertanto, se consideriamo i letti fluviali del Reno e del Savena come potenziali barriere tra le popolazioni del Bolognese, abbiamo 3 aree geografiche: la parte occidentale della provincia, delimitata a est dal corso del Reno (1); la parte centrale della provincia, delimitata a ovest dal corso del Reno e a est da quello del Savena (2); e la parte orientale della provincia, delimitata a ovest dal corso del Savena (3). Dal punto di vista morfologico, i campioni relativi a queste aree presentano differenze significative riguardo al grado di frammentazione delle placche sincipitali (per il quale si osserva un cline da ovest a est) e al numero di gastrostegi e di urostegi (più elevato a ovest, ma soprattutto al centro, e più basso a est).

Nel complesso, le vipere comuni del Bolognese dimostrano una variabilità morfologica ampiamente compresa nei limiti noti in letteratura per la sottospecie francisciredi laurenti, $1768^{7}$. In particolare, esse possiedono caratteristiche intermedie tra quelle degli esemplari dell'Appennino Ligure e dell'Italia centrale (prevalentemente Toscana), rilevandosi tuttavia assai più simili ai secondi che ai primi. Infine, per quanto riguarda alcuni fondamentali aspetti quantitativi, quali il numero dei gastrostegi e quello delle macchie dorsali del tronco, i risultati ottenuti confermano l'esistenza - almeno in Italia - di un gradiente latitudinale (già conosciuto in letteratura): al proposito gli esemplari della provincia di Bologna manifestano diversità altamente significative rispetto a quelli liguri e niente affatto significative rispetto a quelli toscani. 


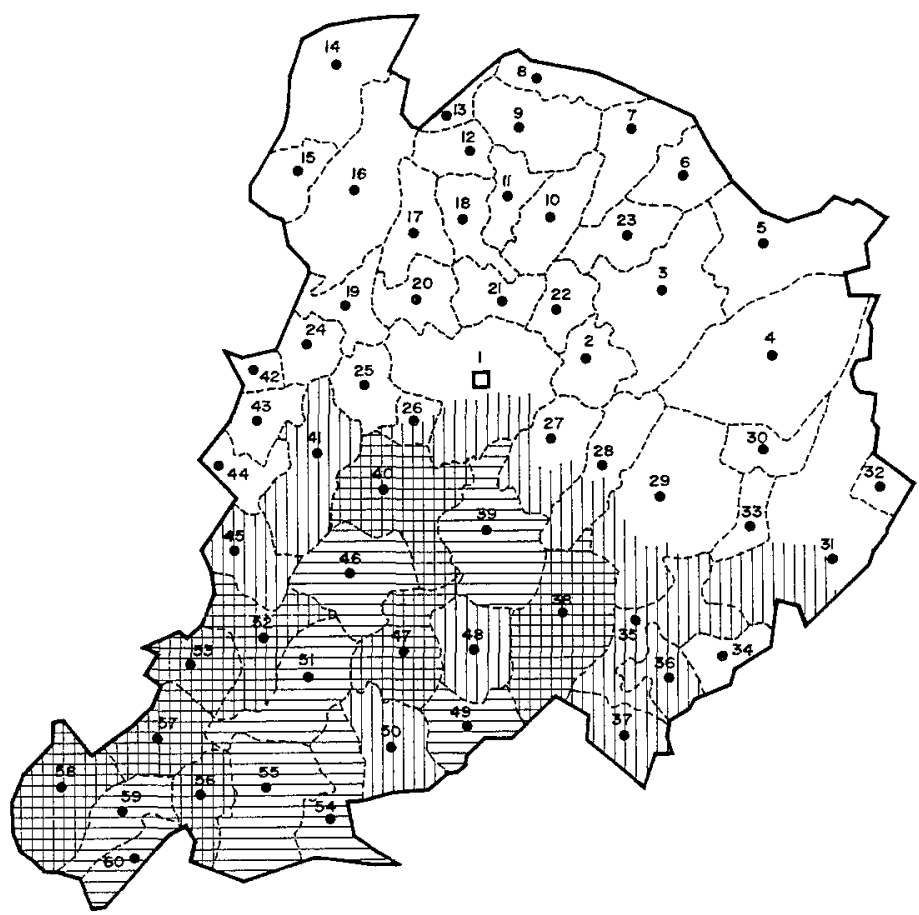

Figura 5.- Distribuzione della vipera comune (Vipera aspis) nella provincia di Bologna sulla base di 4.185 esemplari catturati dal 1966 al 1971 e nel 1973. I territori comunali contrassegnati con la rete rappresentano le zone con il numero di catture da 207 a 635 (dal $4,94 \%$ al $15,17 \%$ ), quelli con le righe orizzontali da 104 a 168 (dal $2,48 \%$ al $4,01 \%$ ) e quelli, infine, con le righe verticali da 1 a 82 (dallo $0,03 \%$ all' $1,96 \%$ ).

I Comuni del Bolognese (le altitudini sono tratte dall'Annuario Generale dei comuni e delle frazioni d'Italia del Touring Club italiano). Bologna, $55 \mathrm{~m}$ (1). Castenaso, $44 \mathrm{~m}$ (2). Budrio, $25 \mathrm{~m}$ (3). Medicina, $25 \mathrm{~m}$ (4). Molinella, $8 \mathrm{~m}$ (5). Baricella, $11 \mathrm{~m}(6)$. Malalbergo, $12 \mathrm{~m}$ (7). Galliera, $16 \mathrm{~m}$ (8). San Pietro in Casale, $17 \mathrm{~m}$ (9). Bentivoglio, $19 \mathrm{~m}$ (10). San Giorgio di Piano, $21 \mathrm{~m}$ (11). Castello d'Argile, $23 \mathrm{~m}$ (12). Pieve di Cento, $14 \mathrm{~m}$ (13). Crevalcore, $20 \mathrm{~m}$ (14). Sant'Agata Bolognese, $21 \mathrm{~m}$ (15). San Giovanni in Persiceto, $21 \mathrm{~m}$ (16). Sala Bolognese, 25 $\mathrm{m}$ (17). Argelato, $21 \mathrm{~m}$ (18). Anzola dell'Emilia, $45 \mathrm{~m}$ (19). Calderara di Reno, $31 \mathrm{~m}$ (20). Castel Maggiore, $27 \mathrm{~m}$ (21). Granarolo dell' Emilia, $28 \mathrm{~m}$ (22). Minerbio, $16 \mathrm{~m}$ (23). Crespellano, $64 \mathrm{~m}$ (24). Zola Pedrosa, $60 \mathrm{~m}$ (25). Casalecchio di Reno, $55 \mathrm{~m}$ (26). San Lazzaro di Savena, $62 \mathrm{~m}$ (27). Ozzano deil'Emilia, $67 \mathrm{~m}$ (28). Castel San Pietro, $75 \mathrm{~m}$ (29). Castel Guelfo di Bologna, $32 \mathrm{~m}$ (30). Imola, $47 \mathrm{~m}$ (31). Mordano, $21 \mathrm{~m}$ (32). Dozza, $190 \mathrm{~m}$ (33). Borgo Tossignano, $102 \mathrm{~m}$ (34). Casalfiumanese, $125 \mathrm{~m}$ (35). Fontanélice, $165 \mathrm{~m}$ (36). Castel del Rio, $215 \mathrm{~m}$ (37). Monterenzio, $206 \mathrm{~m}$ (38). Pianoro, $200 \mathrm{~m}$ (39). Sasso Marconi, $124 \mathrm{~m} \mathrm{(40).}$ Monte San Pietro, $317 \mathrm{~m}$ (41). Bazzano, $113 \mathrm{~m}$ (42). Monteveglio, $280 \mathrm{~m}$ (43). Castello di Serravalle, $320 \mathrm{~m}$ (44). Savigno, $259 \mathrm{~m}$ (45). Marzabotto, $130 \mathrm{~m}$ (46). Monzuno, $621 \mathrm{~m}$ (47). Loiano, $714 \mathrm{~m}$ (48). Monghidoro, $841 \mathrm{~m}$ (49). San Benedetto Val di Sambro, $602 \mathrm{~m} \mathrm{(50).} \mathrm{Grizzana,} 547 \mathrm{~m}$ (51). Vergato, $195 \mathrm{~m}(52)$. Castel d'Aiano, $771 \mathrm{~m}$ (53). Castiglione dei Pépoli, $691 \mathrm{~m}$ (54). Camugnano, $692 \mathrm{~m}$ (55). Castel di Casio, $533 \mathrm{~m}$ (56). Gaggio Montano, $682 \mathrm{~m}$ (57). Lizzano in Belvedere, $640 \mathrm{~m}$ (58). Porretta Terme, $352 \mathrm{~m}$ (59). Granaglione, $69 \mathrm{~m} \mathrm{(60)}$. 
La livrea sfoggiata dalla specie nel Bolognese offre in genere una colorazione di fondo delle parti superiori variabile dal grigio al bruno. Le sopralabiali sono perlopiù bianche. Una barra scura unisce l'occhio alla commessura della bocca e, solo in alcuni maschi, prosegue talvolta lungo i lati del collo per $2-3 \mathrm{~cm}$. In alcuni maschi la macchia scura occipitale a $\mathrm{V}$ rovesciata è fusa con le barre scure postoculari. Il dorso è coperto da numerose barre scure, di solito trasversali e alternate lungo la linea vertebrale o anche unite e allora quasi parallele tra loro. I fianchi mostrano varie macchie scure, di regola rotondeggianti o rettangolareggianti, nei settori non raggiunti dall'ornamentazione dorsale. Le parti inferiori del corpo sono in genere grigie, rosate, giallastre o biancastre, abbondantemente pigmentate di nero.

Gli esemplari esaminati avevano, in media, l'indice staturale compreso tra $58 \mathrm{~cm}$ e $70 \mathrm{~cm}$ circa, 145-147 (maschi) e 148-150 (femmine) ventrali, 40-42 (maschi) e 32-33 (femmine) paia di sottocaudali; inoltre due serie complete di placchette tra l'occhio e le sopralabiali e 21 serie di squame in linea trasversa a metà corpo.

Nel Bolognese, dove è stata trovata da 0 a $1.560 \mathrm{~m}$ di altitudine (in potenza, però, dovrebbe, sia pure occasionalmente, trovarsi anche a quote superiori. Nel Reggiano, per esempio, raggiunge i $1.950 \mathrm{~m}$ s.l.m. sul M. Prado: cfr. già Stagni 1986), la vipera comune predilige le siepi, le piccole macchie e le pietraie più o meno compatte - con ricco contorno di vegetazione erbacea e arbustiva - che si trovano ai margini di coltivi, pascoli e praterie. Il rettile frequenta pure i boschi misti di basse altitudini e i loro margini, le aree periferiche di faggeta cedua con molti ammassi pietrosi, le praterie, le rade boscaglie e i cespuglieti dal piano basale ai limiti inferiori della faggeta e i pascoli di altitudine - talvolta intercalati a bassi arbusti contorti e a pietraie - che si aprono al limite superiore della foresta caducifoglia mista montana.

Cronologia e commenti delle catture di Vipera aspis avvenute in provincia di Bologna dal 1966 al 1971 e nel 1973.

1966. In questa annata furono consegnati 600 serpenti per la determinazione. Di questi 507 furono riconosciuti come vipere comuni. Dei rimanenti, la maggioranza era costituita da bisce d'acqua e una parte da colubri terragnoli in particolare dei generi Coluber e Coronella. Il discreto numero di colubri acquatici catturati è forse attribuibile al motivo che questi, avendo le squame carenate, possono essere scambiati dai profani per vipere soprattutto se sorpresi lontani dall'acqua e in atteggiamenti difensivi che imitano quelli dei viperidi.

Dall'andamento mensile delle catture, risulta che il maggior numero di vipere è stato prelevato da giugno a settembre, e il massimo in agosto (rispettivamente $87,95,150$ e 77 esemplari).

Dalle catture notiamo che le vipere provengono esclusivamente dai settori collinari e montani della provincia dove, a sud della Via Emilia, sembrerebbero - sempre alla luce dei campioni pervenuti- più frequenti nei comuni confinanti con la provincia di Modena (per esempio: 46 vipere a Castel d'Aiano, 1 a S. Lazzaro di Savena e 1 a Castel S. Pietro). 
1967. Nei confronti del 1966 si nota un forte aumento delle catture, con un'incremento di circa il $100 \%$. Infatti, dalle 507 vipere del 1966 si è passati alle 1.103 del 1967. Certamentes ha influito su tale incremento il premio corrisposto dall'APB per ogni serpente velenoso catturato e la maggiore conoscenza della cittadinanza per questa iniziativa, a seguito della campagna di propaganda, sia a mezzo Stampa che attraverso i manifesti e i volantini curati dalla Provincia e dalle associazioni venatorie bolognesi.

Dall' andamento mensile delle catture si può notare che il maggior numero di vipere si registrò da maggio a ottobre, con la punta più alta in settembre (rispettivamente 76, 77, 86, 167, 518 e 104 esemplari).

E' confermata l'ipotesi geografico-ambientale del 1966. Infatti anche quest'anno le località di cattura e di segnalazione sono rappresentate soprattutto dai Comuni in territorio collinare e montano, e la maggiore frequenza dell'ofidio è stata registrata nei Comuni occidentali della provincia di Bologna (per esempio: 212 vipere a Castel d'Aiano, 115 a Lizzano in Belvedere, 2 a Fontanelice e 1 a Ozzano dell'Emilia).

L'individuo segnalato a Bologna e trovato morto alla periferia della città, fu trasportato con molta probabilità dalla montagna con qualche carico di legname.

1968. Numero consistente di vipere catturate anche nel 1968: 1.026. Pertanto si è registrato un certo equilibrio negli ultimi due anni (1967: 1.103 vipere).

Secondo i dati forniti dall'APB, sembra che in alcune località sia stata notata una diminuzione di vipere dopo il lancio di selvaggina stanziale e in particolare di fagiani. In una frazione (San Rocco) del comune di Monzuno, dove furono immessi più francolini di Erckel, pare che dopo la loro introduzione $i$ viperidi non siano stati più segnalati.

Per ciò che riguarda l'andamento mensile delle catture, $\mathrm{i}$ dati confermano quelli degli anni precedenti: periodo più favorevole la primavera-estate con un massimo in agosto (208) e in settembre (191).

Le informazioni geografico-ambientali degli anni precedenti sono ratificate, come anche la maggiore frequenza del Viperidae nel territorio dei comuni montani confinanti con la provincia di Modena (per esempio: 174 vipere a Lizzano in Belvedere, 134 a Castel d'Aiano, 1 a Fontanelice e 1 a Savigno).

1969. Quest'anno sono state consegnate 820 vipere per il riconoscimento.

La diminuzione del rettile, come si può verificare, è più accentuata nei Comuni della zona centrale e orientale dell'Appennino Bolognese (per esempio: 6 vipere a Casalfiumanese, 3 a Castel del Rio, 3 a Fontanelice), mentre per i tre Comuni occidentali dell'alta collina e montagna, confinanti con la provincia di Modena (Castel d'Aiano, Lizzano in Belvedere, Gaggio Montano), i dati quantitativi e percentuali si mantengono costanti (rispettivamente 132,124 e 117 vipere e cioè il $16,09 \%$, il $15,12 \%$ e il $14,27 \%$ ).

Sono confermate le stime relative alle catture mensili con il massimo in settembre (120 in maggio, 73 in giugno, 73 in luglio, 156 in agosto, 214 a settembre e 101 in ottobre). 
1970. Ulteriore diminuzione di vipere nel 1970 rispetto agli anni precedenti. Infatti dalle 820 del 1969 si è scesi alle 503 di quest'anno.

Il rettile è ancora concentrato in due Comuni occidentali (Castel d'Aiano e Lizzano in Belvedere: rispettivamente 110 e 108 vipere) con diminuzione quantitativa ma aumento percentuale (il $21,87 \%$ e il $21,47 \%$ ).

Dai dati che si riferiscono alle catture mensili è confermata anche per il 1970 la perizia già presentata per gli anni passati: concentrazione delle catture nei mesi primaverili ed estivi con massimo in settembre (32 a maggio, 90 a giugno, 61 a luglio, 91 in agosto, 171 a settembre).

1971. Notevoli diminuzioni nelle catture e nelle segnalazioni. Infatti da un minimo di 1 (Bologna-Paderno, Camugnano, Castel d'Aiano, Castel S. Pietro, Grizzana, Marzabotto, Vergato) si arriva a un massimo di 26 (Monterenzio) individui.

Si registra uno spostamento delle aree di maggiore diffusione: dalle zone occidentali della provincia a quelle centrorientali.

L'elevato numero di catture verificatosi negli anni precedenti sembra avere influito sensibilmente sulla consistenza delle popolazioni presenti in tutta la fascia occidentale collinare e montana.

L'andamento mensile delle catture ripete quanto già verificato negli anni passati con il massimo nel mese di settembre (27 a maggio, 18 a giugno, 27 a luglio, 26 in agosto, 37 a settembre).

1973. Diminuzione quantitativa ancora più marcata rispetto a quella dell'anno precedente (appena 57 individui: 22 a Camugnano, da 9 a 1 negli altri territori) e riduzione molto evidente delle aree di cattura, ristrette a pochi Comuni (11 su 60).

Interessante la quasi totale scomparsa dei ritrovamenti nei Comuni della fascia occidentale.

Concentrazione delle catture soprattutto durante i mesi di agosto (12) e di settembre (29).

Considerazioni generali. I dati relativi al complesso dei 7 anni presi in esame, ci consentono una valutazione obbiettiva abbastanza chiarificatrice.

I Comuni della fascia occidentale più alta confinante con la provincia di Modena, sono quelli che hanno consentito le catture e le segnalazioni quantitativamente più consistenti. Invece, quelli che confinano con le provincie di Firenze e di Ravenna, a eccezione di Monterenzio, sembrano avere la più bassa concentrazione di vipere.

Le 4 vipere catturate a Bologna sono: 2 individui raccolti lungo la fascia collinare in località Paderno e altri 2 trovati morti alla periferia della città, dove con molta probabilità erano stati trasportati dalla montagna con qualche carico di legname o di materiale edilizio. Le stesse considerazioni si possono fare per Ozzano dell'Emilia, Imola, San Lazzaro di Savena e Casalecchio di Reno.

Dalle analisi dei dati che si riferiscono alle catture mensili, si può evidenziare che la maggiore concentrazione si verificò in settembre (1.127 esemplari raccolti, pari al $29,56 \%$ del totale). Alta fu anche la quantità di 
vipere comuni prese in agosto (811). Decisamente e progressivamente inferiore quella degli altri mesi: giugno 503, luglio 451, maggio 409 , ottobre 379 , novembre 73 , marzo 70 , gennaio 5-0, febrario 4-0, dicembre 2-0 (fig. $6,7)$.

Come si vede, i mesi estivi, primaverili e autunnali sono quelli che hanno registrato il più alto indice di catture. Ciò corrisponde, evidentemente, al maggiore afflusso di cacciatori e di gitanti nelle località collinari e montane. Infatti, dalle segnalazioni registrate, si è potuto accertare che-trale varie categorie dicittadini, in valore percentuale - detengono il primo posto i cacciatori $(45 \%)$, seguiti da gitanti $(25 \%)$, agricoltori $(10 \%)$, pescatori $(10 \%)$, cercatori di funghi $(8 \%)$ e altri occasionali $(2 \%)$.

Inoltre detti valori, alla luce delle ricerche sulla biologia della specie svolte soprattutto dai francesi Saint Girons (per esempio 1971, 1975, 1978) e Naulleau (p. es. 1966a, b) trovano una chiaraconferma nelle caractteristiche bioclimatiche generali delle aree di raccolta. Infatti, si tratta di territori che rientrano in una fascia di vegetazione submediterranea ${ }^{6}$ perchè gli ambienti collinari - che hanno registrato il massimo delle catture tardoestive e preautunnali, sono ascrivibili al climax dei qurceti a roverella (Quercus

\begin{tabular}{l|c|c|c}
\hline \multicolumn{1}{c|}{ MESE } & NÚMERO VIPERE & PERCENTUALE \% & MEDIA ANNUALE \\
\hline Gennaio & 5 & 0,12 & 0,71 \\
Febbraio & 4 & 0,09 & 0,57 \\
Marzo & 70 & 1,68 & 10,00 \\
Aprile & 241 & 5,76 & 34,42 \\
Maggio & 409 & 9,77 & 58,42 \\
Giugno & 503 & 12,02 & 71,85 \\
Luglio & 451 & 10,77 & 64,42 \\
Agosto & 811 & 19,38 & 115,85 \\
Settembre & 1237 & 29,56 & 176,71 \\
Ottobre & 379 & 9,06 & 54,14 \\
Novembre & 73 & 1,75 & 10,42 \\
Dicembre & 2 & 0,04 & 0,28 \\
\hline Totale & 4185 & 100,00 & 597,85 \\
\hline
\end{tabular}

Figura 6.- Diffusione mensile quantitativa degli esemplari di vipera comune (Vipera aspis) catturati nel Bolognese dal 1966 al 1971 e nel 1973. 


\begin{tabular}{|c|c|c|c|}
\hline COMUNE & NÚMERO VIPERE & PERCENTUALE \% & MEDIA ANNUALE \\
\hline Castel d'Aiano & 635 & 15,17 & 90,71 \\
\hline Lizzano in Belvedere & 554 & 13,24 & 79,14 \\
\hline Gaggio Montano & 395 & 9,43 & 56,42 \\
\hline Vergato & 283 & 6,76 & 40,42 \\
\hline Castel di Casio & 265 & 6,33 & 37,85 \\
\hline Sasso Marconi & 225 & 5,37 & 32,14 \\
\hline Montcrenzio & 222 & 5,30 & 31,71 \\
\hline Monzuno & 207 & 4,94 & 29,57 \\
\hline Pianoro & 168 & 4,01 & 24,00 \\
\hline Marzabotto & 157 & 3,77 & 22,42 \\
\hline Camugnano & 144 & 3,44 & 20,57 \\
\hline Porretta Terme & 140 & 3,34 & 20,00 \\
\hline Grizzana & 136 & 3,25 & 19,42 \\
\hline Granaglione & 118 & 2,81 & 16,85 \\
\hline Monghidoro & 113 & 2,72 & 16,14 \\
\hline Castiglione de Pepoli & 104 & 2,48 & 14,85 \\
\hline Loiano & 82 & 1,96 & 11,71 \\
\hline Castel del Rio & 71 & 1,69 & 10,14 \\
\hline S. Benedetto V.S. & 70 & 1,67 & 10,00 \\
\hline Casalfiumanese & 48 & 1,14 & 6,85 \\
\hline Località imprecisate & 14 & 0,33 & 2,00 \\
\hline Fontanelice & 13 & 0,31 & 1,85 \\
\hline Ozzano dell'Emilia & 7 & 0,17 & 1,00 \\
\hline Bologna & 4 & 0,10 & 0,57 \\
\hline Monte S. Pietro & 4 & 0,10 & 0,57 \\
\hline Imola & 2 & 0,05 & 0,28 \\
\hline Savigno & 1 & 0,03 & 0,14 \\
\hline S. Lazzaro di Savena & 1 & 0,03 & 0,14 \\
\hline Castell S. Pietro & 1 & 0,03 & 0,14 \\
\hline Casalecchio di Reno & 1 & 0,03 & 0,14 \\
\hline Totale & 4185 & 100,00 & 597,85 \\
\hline
\end{tabular}

Figura 7.-Distribuzione quantitativa degli esemplari di vipera comune (Vipera aspis) catturati, nei territori dei 30 Comuni del Bolognese frequentati dal Rettile, dal 1966 al 1971 e nel 1973.

pubescens) e rovere (Quercus petraea), mentre quelli montani che sono stati teatro delle raccolte soprattutto in agosto rientrano nella fascia suboceanica dei faggeti (Fagus sylvatica).

In letteratura la specie è stata indicata anche della pianura Bolognese a nord della Via Emilia. Attualmente questa "arteria" può considerarsi, invece, il limite settentrionale della diffusione di Vipera aspis nella provincia di Bologna (fig. 5). Infatti i boschi planiziari e gli altri ambienti favorevoli, dove un tempo il serpente era confinato, oggi non esistono più: sopravvivono, però, e con essi anche l'ofidio, soprattutto lungo la costiera adriatica, sulla base di osservazioni svolte da alcuni studiosi dal 1969 a oggi. 


\section{Biscia tassellata [Natrix tessellata (Laurenti, 1768)]}

Per Zaffagnini e Bigazzi (1975) in Emilia-Romagna "la colorazione è pressochè uguale a quella della vipera, con macchioline sul dorso più piccole". In base ai nostri dati, il colore di fondo delle parti superiori del corpo è grigio-verdastro o bruno-verdastro. Inoltre: le placche cefaliche possono presentare macchie scure di forma e dimensione irregolari, alla base della nuca c'è una macchia scura a forma di $V$ rovesciata e sia il dorso che i fianchi ostentano tre serie longitudinali di macchie scure, più o meno marcate, disposte a guisa di tasselli alternati (che in alcuni casi e in certi tratti del dorso originano una sorta di zig-zag) e/o in bande quasi trasversali fra loro. Le parti inferiori del corpo, invece, sono bianco-grigiastre, bianco-giallastre o rosate: immacolate (più raramente e di regola solo parzialmente), spruzzate di scuro o coperte di vistose macchie o bande scure.

Per la tassonomia e la corologia classiche le bisce tassellate del Bolognese dovrebbero appartenere alla sottospecie tipo e cioè Natrix tessellata tessellata. Nell' ambito degli esemplari esaminati abbiamo osservato le varietà albo-lineolata (Bonaparte, 1834) [=decipiens (De Betta, 1865), albomaculata (Camerano, 1891)] e viperinoides (Dürigen, 1897) [= flavescens (Werner, 1891)].

"In Emilia-Romagna è comune, [...] è molto legata all'acqua e non l'abbandona quasi mai" (Zaffagnini e Bigazzi 1975).

Nel Bolognose, dove per il momento è stata sorpresa da 0 a $840 \mathrm{~m}$ d'altitudine (fig. 8), vive soprattutto in alvei fluviali e torrentizi a substrato sassoso e roccioso, ma anche in piccole pozze e pantani a fondo sabbiosomelmoso, privi di vegetazione acquatica e situati lungo i margini di aree coltivate e di pascoli con frequenti siepi e modeste macchie.

Alcuni studiosi emiliani e toscani hanno pubblicamente sostenuto che la biscia tassellata sarebbe, a terra, più elegante nei movimenti e più rapida degli ofidi dei generi Coluber ed Elaphe. A parte la stravaganza biologica di proporre che un serpente acquatico e semiacquatico (abituale predatore di animali eterotermi), sia più veloce a terra di ofidi terricoli e arboricoli (attivi predatori di vertebrati eterotermi, ma perlopiù omeotermi), tale opinione è in contrasto con la morfologia, l'ecologia e l'etologia di questi rettili. Infatti tralasciando le non poche differenze morfologiche esterne tra le specie di serpenti appartenenti ai tre generi in oggetto perché ovvie, oltre alla constatazione che Natrix tessellata sia in media la biscia W-paleartica più legata agli ambienti acquatici e altri aspetti eco-etologici perché fin troppo evidentinell'ipotesi che il movimento di un serpente sia approssimabile a un'onda trasversale, la velocità dell'ofidio può essere calcolata come la velocità di propagazione dell'onda, e cioè $V p=\mathrm{fx} \lambda \quad$ ( $V p$ è la velocità di propagazione, $f$ è la frequenza del fenomeno e $\lambda$ è la lunghezza d'onda). Da semplici esperienze visive e/o fisicodinamiche, risulta chiaramente che gli ofidi del genere Natrix, quando serpeggiano, di solito "frenano» l'aria (muso in posizione di "attenti", testa più o meno elevata dal suolo, ampie volute del corpo), mentre gli ofidi dei generi Coluber ed Elaphe 


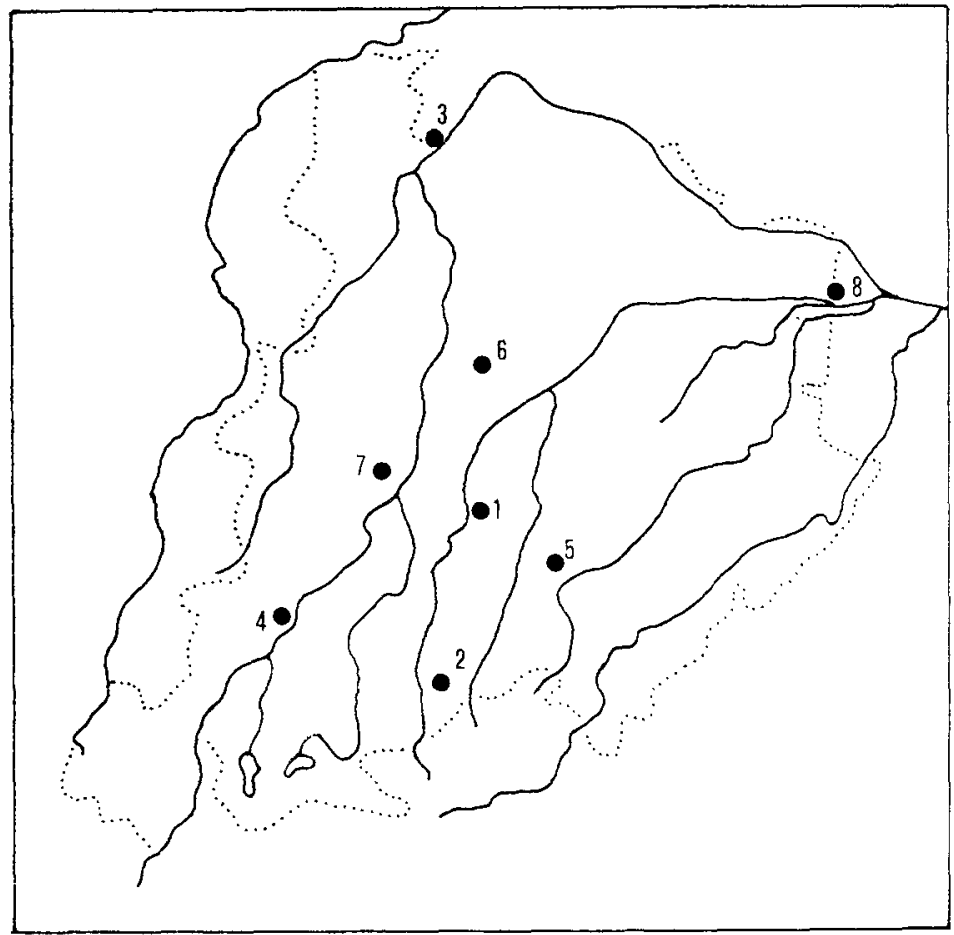

Figura 8.- Distribuzione della biscia tassellata (Natrix tessellata) nella provincia di Bologna:

Zena (Pianoro), $200 \mathrm{~m}$ circa (1), Monghidoro, $841 \mathrm{~m}$ (2), Cento, $17 \mathrm{~m}$ (in realtà si trova nel Ferrarese) (3), Riola (Vergato), $300 \mathrm{~m}$ (4), Monterenzio, 206 m (5), Granarolo dell'Emilia, 28 m (6), Vizzano (Sasso Marconi), 90 m (7).

di regola "fendono" l'aria (muso proteso in avanti, testa più o meno appoggiata al suolo, strette volute del corpo).

\section{Biscia dal collare [Natrix natrix (Linnaeus, 1758)]}

La specie - per Zaffagnini e Bigazzi (1975), che si affidano all'opinione tassonomica mertensiana (Mertens e Wermuth 1960)- in Emilia-Romagna è rappresentata dalle sottospecie helvetica (Lacepède, 1789) e persa (Pallas, 1814), "differenti l'una dall'altra soprattutto per la colorazione [in realtà per la livrea e cioé per il colore e per l'ornamentazione]. Natrix natrix helvetica è grigia, più o meno chiara, con diverse macchie scure che sono disposte regolarmente sopra ed ai lati del dorso: Il giovane ha il collarino dietro la nuca bianco e nero, molto visibile; la parte bianca del collarino si attenua o sparisce, soprattutto nelle femmine, col passare degli anni [...]. Natrix natrix persa è bruno olivacea, più o meno chiara, con sul dorso due linee color crema che partono dalla nuca ed arrivano sino all'apice della coda. I giovani, oltre che per le due linee chiare sul dorso, si distinguono da quelli di Natrix natrix helvetica per il collarino giallo, tendente all'aran- 
cione, invece che bianco; anche in Natrix natrix persa la parte chiara del collare sparisce col passare degli anni".

Sulla base del materiale raccolto e da noi studiato, sebbene la livrea degli esemplari bolognesi rientri -in linea generale - nel sintetico e parziale schema offerto dai predetti studiosi, tuttavia, limitatamente alla colorazione di fondo, la tinta è più variabile in entrambe le razze. Inoltre, sembra che sia forse possibile avanzare una distinzione ornamentale, come accenneremo dopo, a seconda delle caratteristiche bioclimatiche degli ambienti frequentati dal rettile.

Comunque, senza entrare in ulteriori e più tecnici dettagli (sistematici, morfologici, ecologici, ecc.), che d'altra parte sarebbero in contrasto con lo spirito del nostro scritto, ricordiamo soltanto che la variabilità morfologica esterna (qualitativa e quantitativa) di Natrix natrix è stata ed è oggetto di varie ricerche. Secondo quelle di Thorpe (per esempio 1975a,b, 1979, 1980 ), che al momento sembrano godere di maggior credito, le popolazioni della Padania centrale e orientale - e quindi anche del Bolognese- pare che possano rientrare nell'area geografica di possibile introgressione tra $\mathrm{Na}$ trix natrix natrix (in Italia presente almeno in Venezia Giulia e in Friuli) e Natrix natrix helvetica (limitatamente all'Italia continentale dovrebbe essere presente nelle alte pianure e nelle fasce submontane, montane e subalpine del Veneto, Trentino-Alto Adige, Lombardia, Piemonte, Liguria, Emilia orientale e Romagna sudorientale).

In Emilia-Romagna, secondo Zaffagnini e Bigazzi (1975), il fenotipo non lineato sul dorso (e cioè l'attuale var. lanzai Kramer, 1971 del gruppo, o per altri studiosi della sottospecie, helvetica) è "il serpente più comune" (tav. 2), mentre il fenotipo lineato sul dorso [e cioè l'attuale var. persa (Pallas, 1814) del gruppo, o per altri studiosi della sottospecie, natrix) "vive in diversi canali e maceri della bassa Pianura Padana" (tav. 3).

Anche dalle nostre informazioni sembra che helvetica sia più caratteristica negli ambienti dove predominano il cerro (Quercus cerris) e la roverella (Quercus pubesces), mentre persa in quelli dove la farnia (Quercus robur) è stata sostituita da pioppi (Populus alba, P. nigra), salici (Salix alba, ecc). e ontani (Alnus glutinosa).

Comunque, il problema della variabilità morfo-geografica della biscia dal collare offrirà sicuramente ulteriori sorprese. Tra l'altro, come è stato avanzato da più studiosi, è possibile che alcune delle attuali sottospecie siano specie e che più razze, oggi tassonomicamente poco o non considerate, siano invece meritevoli di maggiori attenzioni.

Nel Bolognese (fig. 9), dove per il momento è stata trovata da 0 a 1.420 $\mathrm{m}$ d'altitudine (nel Reggiano, invece, da 0 a $1.850 \mathrm{~m}$ di quota), la specie è molto frequente negli alvei fluviali e torrentizi a substrato sassoso e roccioso, abbastanza comune nei laghi e nelle pozze a substrato sabbioso con ricca vegetazione di idrofite, rara e occasionale nei boschi misti del piano basale, lungo la siepi ai margini dei coltivi e dei pascoli, nelle praterie e nelle rade boscaglie dalle basse altitudini al limite inferiore della faggeta (cfr. già G. Stagni 1986). 


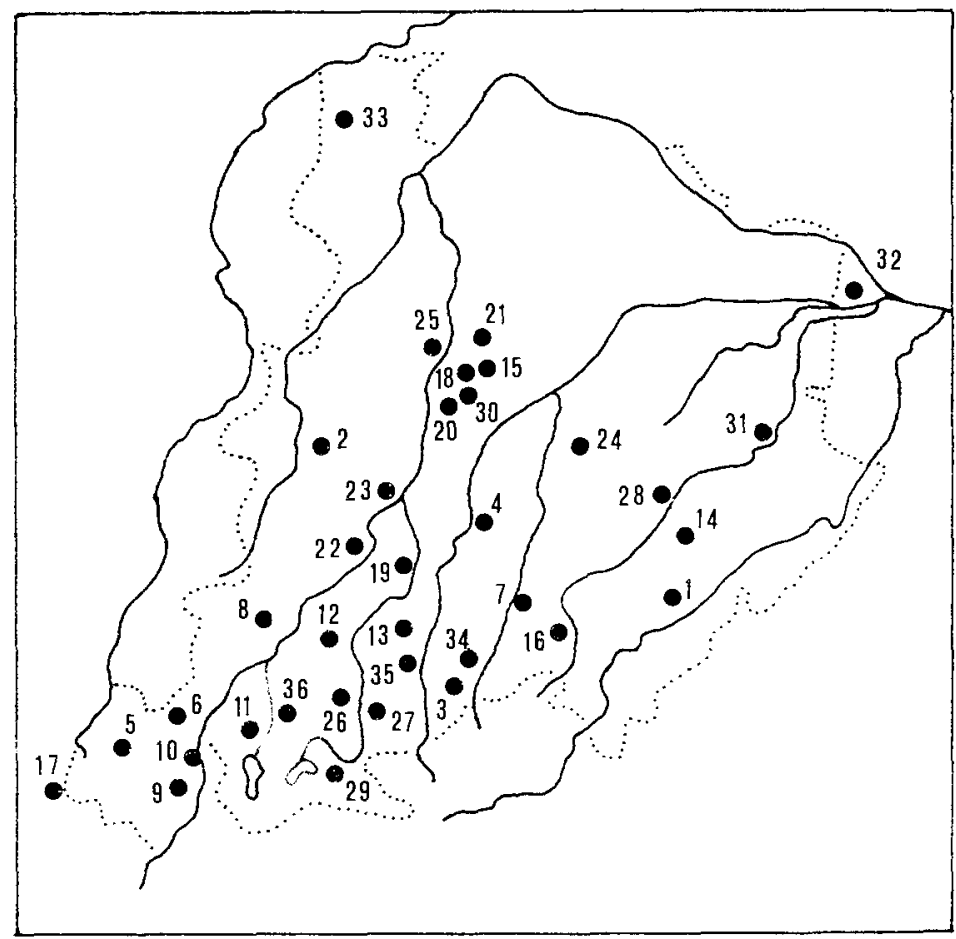

Figura 9.- Distribuzione della biscia dal collare (Natrix natrix) nella provincia di Bologna: Casalfiumanese, $100 \mathrm{~m}$ circa (1), Monte San Pietro, $317 \mathrm{~m}$ (2), Monghidoro, $841 \mathrm{~m}$ (3), Pianoro, 200-450 m (4), Lizzano in Belvedere (comprese le località Madonna dell'Acero e Rifugio Cavone), 604-1.420 m (5), Gággio Montano, $682 \mathrm{~m}$ (6), Monterenzio, $206 \mathrm{~m}$ (7), Riola di Vergato, $810 \mathrm{~m}$ (8), Granaglione, $450 \mathrm{~m}$ (9), Porretta Terme (in altitudine fino al M. Piella), 349-1.119 m (10), Castel di Casio, $533 \mathrm{~m}$ (11), Grizzana (Monte Stanco), $600 \mathrm{~m}$ circa (12), Monzuno, $625 \mathrm{~m}$ (13), Montecatone, $242 \mathrm{~m}$ (14), San Lazzaro di Savena, $60 \mathrm{~m}$ circa (15), Villa Sassonero, $434 \mathrm{~m}$ (16), Lago Scaffaiolo, $1775 \mathrm{~m}$, in provincia di Modena (17), Quarto Superiore, $35 \mathrm{~m}$ circa (18), Vado, $157 \mathrm{~m}$ (19), Casalecchio di Reno, $70 \mathrm{~m}$ circa (20), Torreverde-Castel Maggiore, $22 \mathrm{~m}$ (21), Marzabotto, $130 \mathrm{~m}$ (22), Sasso Marconi (anche Bedolo e M. Mario), 124-550 m (23), Ozzano Emilia, 67 m (24), Calderara di Reno, $30 \mathrm{~m}$ (25), Lagaro, $393 \mathrm{~m}$ (26), Sant'Andrea Valle di Sambro, $600 \mathrm{~m}$ circa (27), Castel San Pietro Terme, $75 \mathrm{~m}$ (28), Castiglione dei Pepoli, 691 $\mathrm{m}$ (29), Monte Donato, $200 \mathrm{~m}$ circa (30), lungo il torrente Sillaro presso Sesto

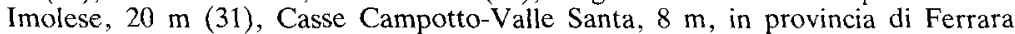
(32), Crevalcore, $20 \mathrm{~m}$ (33), Roncastaldo (Loiano), $600 \mathrm{~m}$ (34), Castel dell'Alpi (San Benedetto Val di Sambro), $750 \mathrm{~m}$ (35), Torrente Limentra di Treppio (Camugnano), $510 \mathrm{~m} \mathrm{(36).}$ 


\section{Biscia viperina [Natrix maura (Linnaeus, 1758)]}

Zaffagnini e Bigazzi (1975) non ricordano Natrix maura, né per il Bolognese né per la regione, perché "all'Istituto di Zoologia dell'Università di Bologna non sono noti reperti per poter considerare" questa specie presente "in Emilia e Romagna". In verità, la presenza della biscia viperina nella regione -dove era stata già documentata, per esempio, nel 1887 e nel 1957- in questi ultimi anni ha trovato ulteriori conforme grazie alle solerti ricerche di alcuni naturalisti (fig. 10).

A prima vista, o a un esame superficiale, questa biscia può essere confusa con la tassellata o, forse più raramente, con quella dal collare. In sviste del genere sono incorsi - "thecretic bias" a parte- erpetologi, zoologi e naturalisti, sia dilettanti che professionisti, e queste valutazioni, svolte su esemplari vivi o morti, potrebbero essere uno dei motivi per cui "Natrix viperina" sia, a tutt'oggi, considerata rara in Emilia e nella Lombardia occidentale.

Secondo le esperienze di alcuni biologi emiliani e lombardi, pare che la biscia viperina sia piuttosto localizzata nelle valli in cui vive, dove sarebbe rappresentata da modeste colonie che frequentano aree geografiche più o meno abitate anche dalle due altre specie di bisce d'acqua.

Certi autori ritengono che la biscia viperina sia, in Europa, la specie più acquatica del genere Natrix; altri, invece, sostengono che questo primato spetta alla biscia tassellata. A nostro avviso si tratta di affermazioni generiche e opinabili perché legate a troppi fattori biologici: le caratteristiche trofiche e climatiche degli ambienti frequentati dagli ofidi, le condizioni fisiologiche, l'età, il sesso, il periodo di attività delle bisce, ecc. Senz'altro, in determinate circostanze, Natrix maura è più acquatica di Natrix tessellata, ma è vero anche il contrario. Forse sarebbe più oggettivo sposare la tesi degli studiosi che presentano la biscia viperina come la più eclettica fra le tre specie di bisce d'acqua europee e le attribuiscono caratteri eco-etologici sia peculiari sia simili a quelli della biscia tassellata. Questa valutazione potrebbe spiegare, tra l'altro, l'indubbio vantaggio ecologico di Natrix maura nei riguardi di Natrix natrix nelle regioni geografiche dove le due specie convivono. Una ricerca sulla biologia di Natrix maura, Natrix natrix e Natrix tessellata in una "stazione" dove le bisce coabitano sarebbe molto interessante.

La rarrefazione di Natrix tessellata a nord della fascia di vegetazione alpica e a ovest della Selva Boema, la sua localizzazione (naturale o antropica?) nell'Europa centrale e la sua sostituzione (netta e/o graduale), nelle Alpi sud-occidentali e nel Bassopiano Francese, con Natrix maura, ricordano le ipotesi che la biscia viperina abbia soppiantato la biscia tassellata nell'Europa occidentale, che qui si sia "impossessata" della nicchia ecologica occupata a est da Natrix tessellata e che svolga, in relazione alle altre due bisce, un ruolo ecologico simile a quello attribuito a Elaphe scalaris rispetto agli Elaphe longissima, quatuorlineata e situla. 


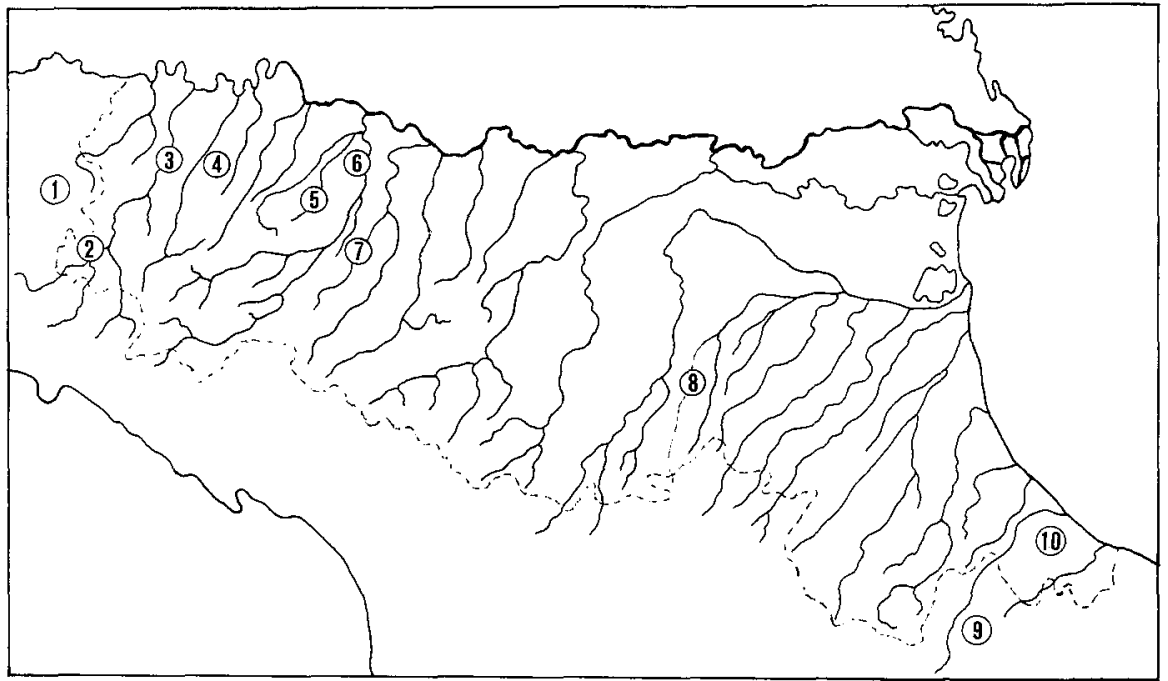

Figura 10.- Distribuzione, parziale e approssimativa, reale e ipotetica, della biscia viperina (Natrix maura) in Emilia-Romagna e immediati dintorni.

Val Staffora: Rio Val Crosa, $550 \mathrm{~m}$ circa, $180-200 \mathrm{~m}$ prima della sua confluenza con il torrente Lella (San Michele, Varzi) (Marco Zuffi di Milano in litteris 24 febbraio 1983) (1). Ponte Organasco nell'alta Val Trebbia, $500 \mathrm{~m}$ circa (Sergio Mezzadri di San Nicolò, Piacenza, in più occasioni sia in litteris che in verbis) (2). In un pozzo di irrigazione situato presso il fiume Trebbia in frazione Cà Buschi (Rivergaro, Piacenza), $100 \mathrm{~m}$ circa (Stefano Zilocchi di Piacenza in litteris 5 gennaio 1987 (3). Torrano in Val di Nure (Piacenza), $140 \mathrm{~m}$ circa. Secondo Schätti (1982) l'esemplare emiliano segnalato da Lugaro (1957) di "Toviano Piacentino (Henryzipperlen 1931)" - ma in realtà etichettato "Castello di Toviano (Piacenza), leg. Henry Zipperlen, IV. 1931" (Zuffi 1983)- potrebbe provenire da Torrano. Tuttavia, per Zuffi (l.c.) questa località "introvabile sugli annuari del TCI" —e non ravvisata neanche da Lanza (1983) - é "piuttosto da identificarsi con Tabiano Bagni [...] o, più probabilmente, con Tabiano Castello". Riteniamo che sia più attendibile l'ipotesi di Schätti (l.c.), sia perché i due Tabiano si trovano in provincia di Parma invece che in provincia di Piacenza, sia perché a Torrano c'è una località chiamata "Castello" dall' edificio omonimo che la caratterizza (cfr. anche Rubbi e Clò 1980) (4). Tabiano Castello $(7,5 \mathrm{~km}$ a sud di Salsomaggiore Terme, Parma), $300 \mathrm{~m}$ circa. Per Zuffi, (l.c.) potrebbe essere questa la località di provenienza dell'individuo segnalato da Lugaro (l.c.) (5) Dintorni di San Secondo Parmense, $35 \mathrm{~m}$ circa, 1 giovane, legit Adolfo Fiori di Roma il 29 settembre 1989 e donavit al Parco Nazionale d'Abruzzo il 14 ottobre 1989 (6). Nelle colline di San Vitale di Baganza (Parma), 300-400 m circa (Del Prato 1887, 1899, de Marchi 1974, 1980) (7). Santa Maria di Zena (Pianoro, Bologna) in località Fazzola, 1 giovane, legit Ettore Negrini di Santa Maria di Zena il 10 maggio 1967, consegnato il 12 maggio 1967 dall'Amministrzione Provinciale di Bologna al Laboratorio di Zoologia Applicata alla Caccia (8). Carpegna (Pesaro), l'abitato è a $748 \mathrm{~m}$ : un esemplare catturato da "Ottorno" [in realtà Ottorino] Hoffer di Rimini (Silvestri 1972) (9). Presso il torrente Ausa [nel 1978 l'autore scrive "Ansa"] (Forli): un esemplare catturato da Ottorino Hoffer di Rimini (Silvestri 1.c.) (10). Queste due ultime segnalazioni, in particolare, devono essere confermate. Nelle "stazioni" 6 e 8 riteniamo che, fino a prova contraria, la specie sia arrivata indirettamente e senta acelimatazione. 


\section{Colubro leopardino [Elaphe situla (Linnaeus, 1758)]}

Silvestri (1972) scrive che il signor Ottorino Hoffer di Rimini, a cui deve buona parte delle inedite notizie erpetologiche che riporta, trovò "due esemplari" di questa specie "uno in prossimità della sua abitazione - frazione di S. Martino in Venti del Comune di Rimini- e l'altro nel Rio Marano". L'autore aggiunge, inoltre, che a quanto gli "costa questa sarebbe l'unica segnalazione in Romagna. Ottorino Hoffer assicura tuttavia che il caratteristico disegno delle macchie del corpo e in particolare della nuca, non lo possono avere indotto in errore. Anche in questo caso, come per la Natrix maura sarebbe indispensabile la conservazione dei soggetti rinvenuti".

Zaffagnini e Bigazzi (1975) ritengono che non siano "noti reperti per poter considerare" il colubro "presente in Emilia e Romagna". Silvestri (1978) ripete quanto giá comunicato nel 1972 e aggiunge che, secondo quanto gli scrisse in proposito l'erpetologo e zoologo fiorentino B. Lanza, "probabilmente il colubro leopardino era stato confuso con il colubro di Ricccioli".

Forse è possibile scambiare Coronella girondica per Elaphe situla e viceversa, ma certamente è un abbaglio piuttosto improbabile anche per un dilettante. Il "caratteristico disegno delle macchie del corpo e in particolare della nuca" (tav. 6), indicato dall'appassionato riminese per la determinazione dei due esemplari in causa, sono qualità ornamentali molto più distintive dell' Elaphe che non della Coronella girondica. Inoltre gli aspetti bioclimatici delle due aree geografiche in oggetto oggi non sembrano più conformi alle esigenze ecologiche di Coronella girondica.

L'esperienza ci ha insegnato che l'ofidio italiano, più comunemente confuso con il colubro leopardino, è il cervone giovane (tav. 7) ${ }^{8}$. Dal momento che alcuni zoogeografi suppongono che, per motivi bioclimatici, Elaphe quatuorlineata non dovrebbe essere presente, allo stato naturales, a nord della Val Foglia, potrebbe trattarsi di un ennesimo caso di introduzione? Non dimentichiamoci, infatti, che tra Rimini e Ancona si trovano le basi operative dei più attivi rettilari della Riviera Adriatica.

Comunque, il colubro leopardino - che in Italia vivrebbe in Puglia, Basilicata, Calabria ${ }^{9}$ e Sicilia - non ci risulta che, sul versante adriatico della sua distribuzione italiana, sia stato trovato nella Valle del Fortore (Puglia settentrionale) o in quella del Saccione (Molise orientale), dove potenzialmente, però, potrebbe essere presente (soprattutto in località Torre Fantine-Ramitelli).

Tuttavia, "Herr Dr. Rabenhorst fand auf den Abruzzen auch eine prächtige gestreifte Varietät von einem Männchen des Coluber leopardinus" che, nel 1856, l'erpetologo Dehne di Dresda (Allgem. deustch, naturhist. $Z$., vol. 2, N.S., pag. 218). descrisse come var. striata perché, tra l'altro (pag. 219), "man schlange z. B. Schin's Amphibien Tab. 58 nach, wo ein gestreifter Tropidonotus Natrix abgebildet ist". Questa notizia, pubblicizzata da Mertens e Wermuth (1980) per motivi sinonimici, fu ripresa, dubbitativamente, da molti studiosi per ragioni geonemiche. Noi riteniamo che, 
sotto quest'ultimo punto di vista, sia infondata. Lo studioso di Dresda, infatti, scrive (pag. 219), oltre al resto, che ebbe "Von den Abruzzen" anche un esemplare di "Tyria dahlii Michahelles, Fitzinger", oggi Coluber najadum (Eichwald, 1831), sebbene "Mein Exemplar, welches aber nicht aus Italien, sondern aus Dalmatien stammt, ...". Ci sembra, quindi, molto verosimile che sia il colubro leopardino che il colubro saetta (quest'ultimo assente in Italia) provenissero dalla Dalmazia e che il primo per errore sia stato assegnato all'Abruzzo, regione visitata, per motivi anche naturalistici, dal racoglitore (il dr. Rabenhorst).

\section{Cervone [Elaphe quatuorlineata (Lacepède, 1789)]}

Questo serpente è indigeno in Italia soltanto nelle regioni meridionali (Sicilia compresa) e centrali: a nord fino alla valle del Serchio (Toscana), ma forse anche a quella del Magra, e al Montefeltro (soprattutto marchigiano) dove probabilmente non si spinge a settentrione dell'area Foglia-Marecchia.

Dal punto di vista ecologico, il cervone è, almeno nella penisola Italica e in quella Ellenica, un serpente tipico della regione bioclimatica xeroteri$\mathrm{ca}$, dove vive soprattutto nella bassa boscaglia sempreverde (ambiente mediterraneo-arido, in cui non è abituale), nella foresta sempreverde di clima temperato-caldo (ambiente mediterraneo) e nella foresta caducifoglia mista con dominanza di Quercus (ambiente collino-planiziare). Questo rettile, sebbene sia caratteristico del piano basale della zona di vegetazione mediteranea e quindi delle sottoregioni bioclimatiche termo-meso-submediterranea, si spinge talvolta, attraverso le valli o sui versanti più termofili della fascia di vegetazione sannitica, fino a 900-1.200 m d'altitudine.

Da queste sintetiche premesse è evidente che, mentre il limite italiano nord-occidentale della distribuzione naturale della specie è facilmente identificabile, quello nord-orientale è invece potenziale. Infatti, fermo restando che per motivi ecologici il cervone non dovrebbe essere autoctono nella $\mathrm{Pa}$ dania - e cioè in un'area di clima temperato della regione bioclimatica mesaxerica - i limiti adriatici sud-orientali della fascia di vegetazione medioeuropea della zona omonima, che caratterizza quest'area geografica, non sono netti: invero tale fascia penetra ampiamente lungo il piano basale fino alle Marche, dove sfuma progressivamente verso la fascia sannitica della zona di vegetazione mediterranea.

Il cervone, tuttavia, è stato segnalato di alcune località della zona di vegetazione medioeuropea, ma è anche nostra opinione che si tratta di individui importati $o$, in alcuni casi, di notizie incerte (fig. 11). In questi ultimi 12 anni, l'Italia è stata teatro di "scoop" ofidiologici presentati dagli autori come incredibili reperti biogeografici, ma ritenuti da molti altri studiosi - fino a prova contraria - ennesimi casi di introduzione (cf. per esempio Bruno e Maugeri 1990). 


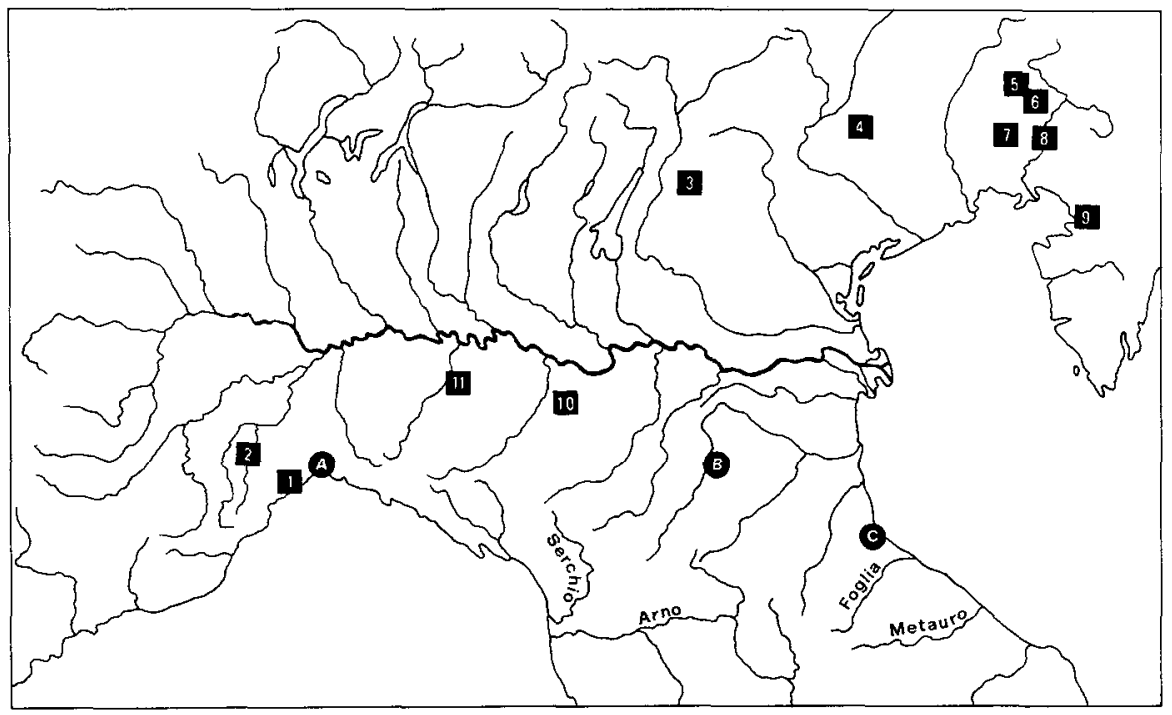

Figura 11.-Alcune "stazioni" dell'Italia settentrionale dove è stato segnalato il cervone (Elaphe quatuorlineata). Località certe (quadrati) e dubbie (cerchi). Le notizie prive di referenza si riferiscono alla nota di S. Bruno (Studi trent. Sci. nat., Trento, ser. B, vol. 43, fasc. 2, pag. 202, 1966). La specie potrebbe essersi acclimatata nelle stazioni 7 e 11 .

Liguria: M. Beigua, $1.280 \mathrm{~m}$ circa (1). Piemonte: Cissone (Monforte d'Alba), $700 \mathrm{~m}$ circa (2). Veneto: Maso in Val Posina (e non in Val di Ledro come è scritto erroneamente nell'originale), $700 \mathrm{~m}$ circa (3). Veneto: Bosco del Cansiglio (4). Friuli: Montefosca (Udine), $725 \mathrm{~m} \mathrm{(5).} \mathrm{Friuli:} \mathrm{Cepletischis} \mathrm{(Udine),}$ $560 \mathrm{~m}$ (6). Friuli: San Giovanni al Natisone (Udine), $50 \mathrm{~m}$ circa (L. Lapini, 1983. Anfibi e Rettili. Lorenzini, Udine, pp. 122-123) (7). Friuli: M. Sabotino (Gorizia), $600 \mathrm{~m}(8)$. Venezia Giulia: M. Carso (Trieste), $300 \mathrm{~m}$ circa (9). Emilia-Romagna: il 30 giugno 1977 un adulto, in parte schiacciato, sarebbe stato raccolto in un campo di frumento in località Casello Poldi (Molétolo, Parma), $43 \mathrm{~m}, \mathrm{e}$ portato all'Istituto di Zoologia dell'Università il 24 agosto 1977 dall'erpetologo e naturalista parmense Manuel Mongini). Per la provincia di Parma, forse anche in base a detta notizia, il cervone è segnalato da de Marchi (1980), sebbene l'autore sostenga (pag. 148) -ignoriamo le sue fonti- che la specie "predilige le zone acquitrinose delle montagne" (10). Emilia-Romagna: una "small population" fu scoperta nei dintorni di Ponte d'Olio (Piacenza), $215 \mathrm{~m}$ circa, a partire dal 1976 (Niekisch 1982) (11). Liguria: provincia di Genova (Sassi 1846, De Betta 1874). E' possiblile che l'autore abbia confuso il cervone con uno o più colubri d'Esculapio a livrea lineata? (A). Emilia-Romagna: dintorni di Bologna. Questa stazione è ricordata da più studiosi tra i quali, per esempio, Duméril e Bibron (1854) - che ebbero il cervone da C. Ranzani, professore di zoologia all'Università di Bologna-, Günther (1858), De Betta (1868, 1874), Camerano (1891), Boulenger (1894) (B). Emilia-Romagna: San Martino in Venti e Rio Marano (dintorni di Rimini). Silvestri $(1972,1978)$ lo segnala, indirettamente, prima come colubro leopardino e poi ipotizza, ancora indirettamente, che possa essere un colubro di Ricciòli. Nell'ambito delle ipotesi, noi supponiamo che i due serpenti in oggetto potessero essere giovani cervoni (C).

In fase di stampa precisiamo che M. Mogini, prima dell'ottobre 1992, fece sapere a G. Stagni che il cervone di Casello Pololi era in realtà u colubro d'Escuapio. Pertanto la "stazione" 10 deve essere cassata. 


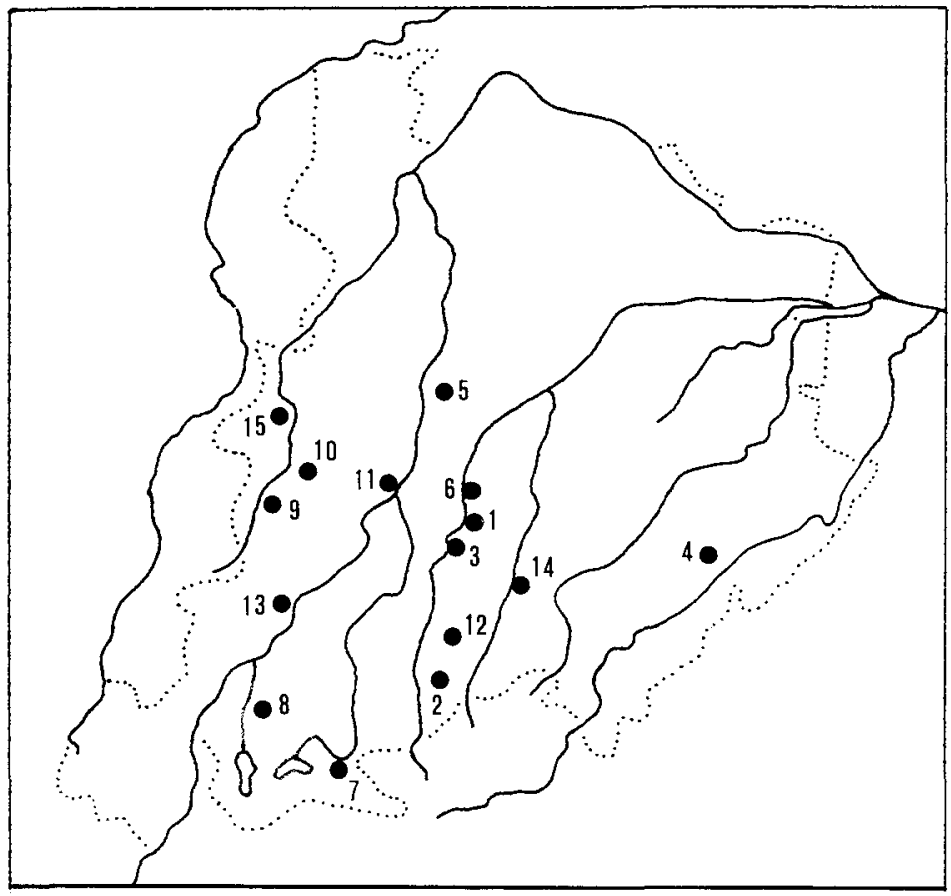

Figura 12.- Distribuzione del colubro di Esculapio (Elaphe Iongissima) in provincia di Bologna. In letteratura la specie è segnalata di alcune località dove oggi sembra scomparsa o comunque da noi non ritrovata.

Pianoro (Carteria di Sesto, Zena, Zula), 120-350 m (1), Monghidoro, 700$840 \mathrm{~m} \mathrm{(2),} \mathrm{Livergnano,} 500 \mathrm{~m}$ circa (3), Pezzuolo Casalfiumanese, $150 \mathrm{~m}$ circa (4), Casalecchio di Reno, 70-100 m (5), Pieve del Pino, $165 \mathrm{~m}$ circa (6), Castiglione dei Pepoli, $690 \mathrm{~m}$ circa (7), Castrola (Camugnano), 400 circa (8), Savigno (Cà Bertolani), $650 \mathrm{~m}$ (9), Monte San Pietro (M. Bonsara), $500 \mathrm{~m}$ circa (10), Sasso Marconi (Bodolo, Prati di Mugnano), 250-350 m (11), Loiano (La Guardia), 600 $\mathrm{m}$ (12), Vergato (Riola), $250 \mathrm{~m}$ (13), Monterenzio (Castelnuovo di Bisano), 420 $\mathrm{m}$ (14), Monteveglio, $115 \mathrm{~m}$ circa (15).

\section{Colubro d'Esculapio [Elaphe longissima (Laurenti, 1768)]}

In Emilia-Romagna il colore degli adulti varierebbe "da olivastro a nero" sul dorso e sarebbe "giallo sul ventre" (Zaffagnini e Bigazzi 1975). Secondo i nostri dati le livree di base variano, nelle parti superiori del corpo, dal giallastro al bruno-giallastro e al verde oliva e, nelle parti inferiori, dal giallastro al bianco-giallastro e (più raramente) al grigio-giallastro. Il dorso è ornato in genere da minuscole macchioline chiare; in alcuni casi anche da 4 fasce scure longitudinali e parallele o, più di rado, da una fascia chiara, mediana e longitudinale.

Le popolazioni del Bolognese, almeno sulla base dei loro caratteri morfologici qualitativi ${ }^{10}$, rientrano nella variabilità della sottospecie tipo e cioè di Elaphe longissima longissima. 
In Emilia-Romagna, il colubro d'Esculapio "è abbastanza comune" (Zaffagnini e Bigazzi 1975). Elaphe longissima è serpente soprattutto termofilo e mesofilo: pertanto, in provincia di Bologna (fig. 12), dovrebbe potenzialmente vivere sia in pianura (rive e greti fluviali, ruderi, ecc.) che in collina (querceti mesofili e loro margini, boschi e boscaglie di carpino nero e di orniello, castegneti, boschi misti di roverella e di cerro, cespuglieti xerofili, garighe, boschi ripariali, leccete relitte, ecc.), ma anche in montagna (pascoli, boscaglie e boschi di faggio). In realtà (cfr. già Stagni 1986), il rettile frequenta abitualmente sia $\mathrm{i}$ boschi misti di pianura e di collina e $\mathrm{i}$ loro margini che i confini ricchi di siepi e di modeste macchie dei coltivi e dei pascoli; saltuariamente vive anche nelle praterie e nelle rade boscaglie dal piano basale fino ai limiti inferiori della faggeta $(840 \mathrm{~m}$ circa d'altitudine).

\section{Colubro di Ricciòli [Coronella girondica (Daudin, 1803)]}

Gli ambienti naturali di questo serpente - elemento faunistico mediteraneo occidentale, a corologia europea sudoccidentale-mauretanica di tipo ridotto (Baleari, Corsica e Sardegna escluse; forse assente nelle porzioni occidentali e orientali della sua distribuzione maghrebina) - sembrano piuttosto singolari se paragonati a quelli degli altri ofidi europei e mediterranei. Limitandoci all'Italia, è stato rilevato che, almeno in potenza, le popolazioni continentali o cisalpine dovrebbero vivere nella fascia di vegetazione medioeuropea (boscaglia e foresta di caducifoglie miste con dominanza di Quercus), mentre quelle peninsulari o appenniniche dovrebbero frequentare le fasce di vegetazione mediterranea (boscaglia e foresta sempreverdi di clima temperato-caldo) e sannitica (tipo di vegetazione simile a quello della fascia medioeuropea dell'omonima zona vegetazionale.

Oggi — dopo che l'intervento dell'uomo ha praticamente sostituito ai boschi e alle boscaglie miste caducifoglie della Padania (in questo caso soprattutto centrale e occidentale) una steppa a cereali con isole di aggruppamenti vegetali spontanei contaminati da piante avventizie- il colubro di Ricciòli è accidentale in questo ambiente collino-planiziare (dove, forse anche alla fine dell'Ottocento e nei primi trent'anni del Novecento, poteva essere già localizzato, ma forse non raro) e sembra vivere esclusivamente nelle aree con vegetazione extrazonale e xerica di tipo mediterraneo. Più comune è invece nella zona di vegetazione mediterranea, dove però -e questa è considerata una delle sue originalità ecologiche- il rettile frequenta soprattutto le gariche, i pascoli e gli incolti aridi e temperato-caldi dove fioriscono gli asfodeli fistulosus e microcarpus, e le colline coperte di boscaglie e di boschi termofili di latifoglie decidue (in particolare le zone del Quercus pubescentis e del Cercis siliquastrum).

Nel Bolognese, dove al momento ci risulta più comune del colubro liscio (fig. 13), Coronella girondica è stata sorpresa da 0 a $840 \mathrm{~m}$ d'altitudine: lungo i margini e nei boschi misti di querce e di carpini del piano basale, nelle praterie e nelle rade boscaglie dei piani basale e collinare fino ai limiti inferiori della faggeta e su emergenze rocciose. 


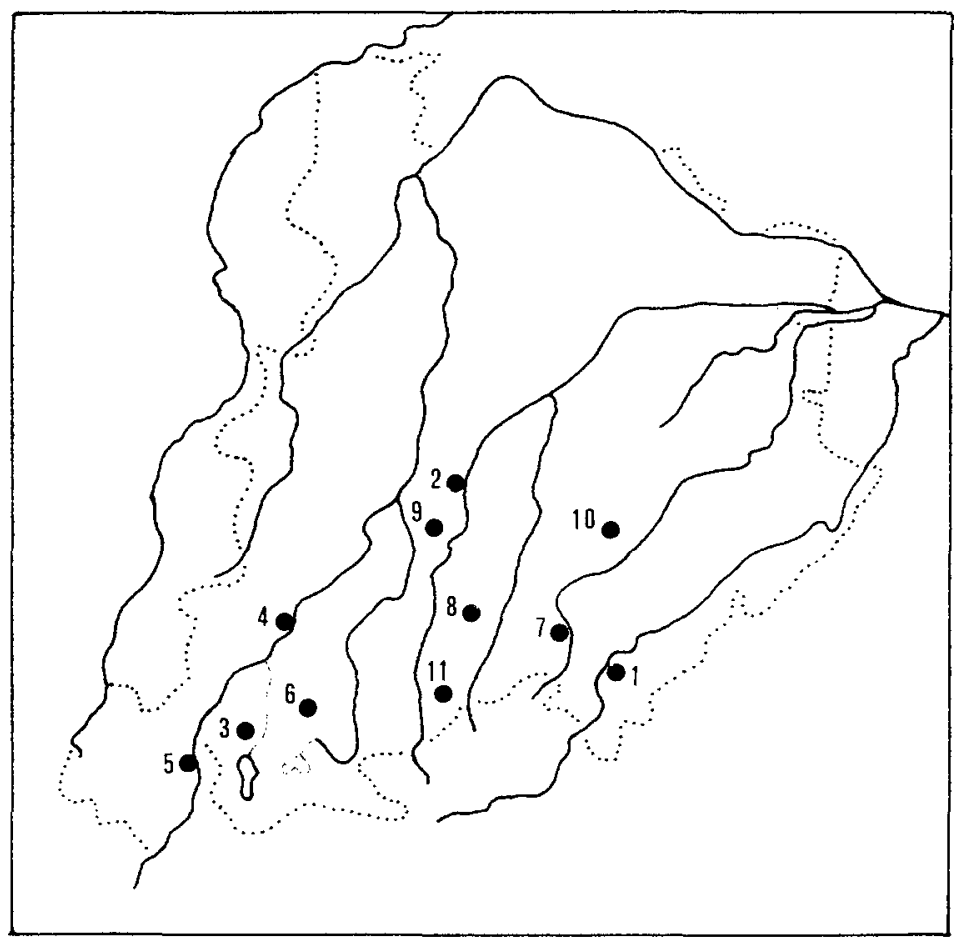

Figura 13.- Distribuzione del colubro di Ricciòli (Coronella girondica) nella provincia di Bologna. La specie è stata segnalata, anni or sono, anche della Padania, dove però non ci risulta che sia stata ritrovata.

Gaggio Montano, $682 \mathrm{~m}$ (1), Pianoro, 200-550 m (2), Castel di Casio, $533 \mathrm{~m}$ (3), Vergato, $195 \mathrm{~m}$ (4), Porretta Terme, $352 \mathrm{~m} \mathrm{(5),} \mathrm{Camugnano,} 692 \mathrm{~m}$ (6), Villa Sassonero, $435 \mathrm{~m}$ circa (7), Selva di Sabbioni-Loiano, $700 \mathrm{~m}$ circa (8), Monte Adone, Bodolo (Sasso Marconi), 350-600 m (9). Farneto, $466 \mathrm{~m}$ (10), Monghidoro, $840 \mathrm{~m}(11))$.

Il reperto di Bassi (1989), contrariamente al titolo della sua nota, non riguarda l'Emilia-Romagna e neppure, come recita la didascalia della figura, l'Appennino Imolese, bensì il territorio di Firenzuola e quindi l'Appennino Fiorentino (Toscana).

\section{Colubro liscio (Coronella austriaca) Laurenti, 1768}

In provincia di Bologna, quest'ofidio sembra raro e localizzato. "Molto raro" lo considerano Zaffagnini e Bigazzi (1975) in Emilia-Romagna. Senni (1985) scrive che nel Ravennate si trova "con scarsa frequenza". Stagni (1986) lo trovò soltanto in una località della provincia di Bologna e in cinque località del Reggiano: in quest'ultima provincia, però, Bertusi (1982), sebbene lo segnali di tre località, ritiene che il colubro sia comune tanto in 


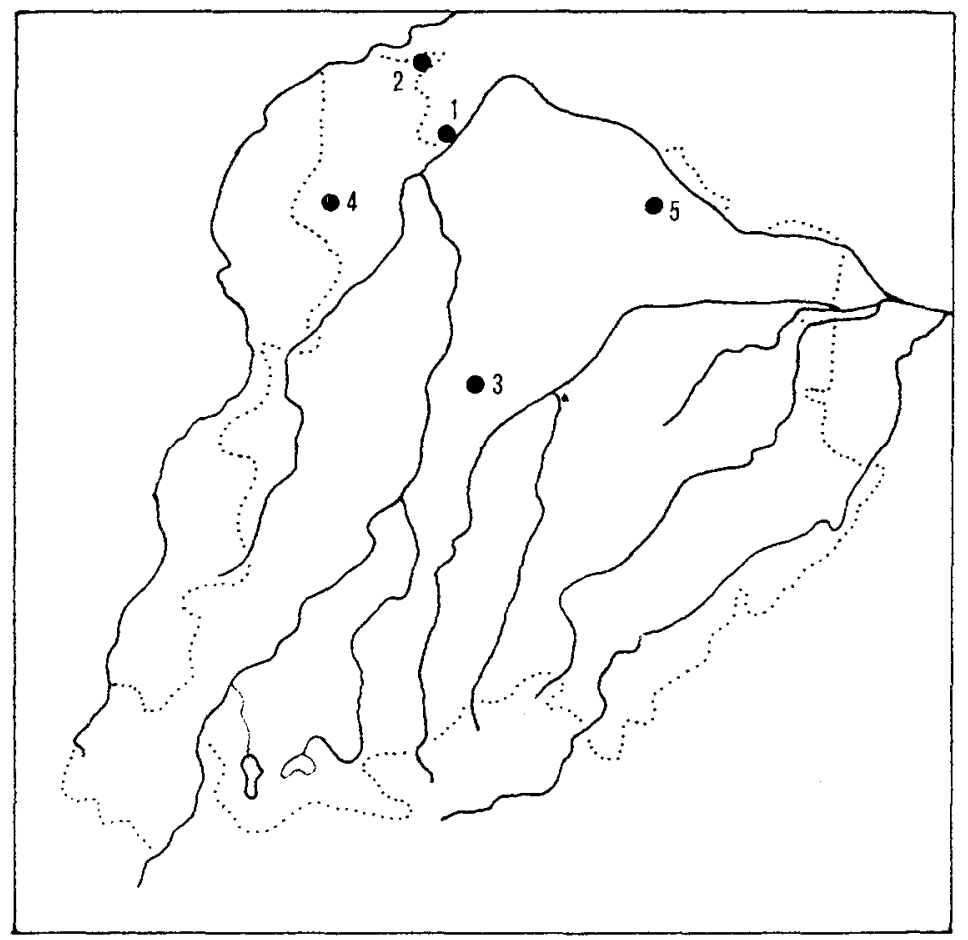

Figura 14.- Distribuzione del colubro liscio (Coronella austriaca) nella provincia di Bologna. Sulla frequenza della specie in Emilia-Romagna i pareri degli studiosi sembrano alquanto discordi. Indipendentemente dal fatto che la sua diffusione possa variare da provincia a provincia (a seconda delle caratteristique bioclimatiche delle zone, in base al numero dei ricercatori e alla loro esperienza "di campagna", ecc.), nel Bolognese il rettile pare al momento abbastanza occasionale e meno raro a nord della Via Emilia.

FF.SS. San Donato, $50 \mathrm{~m}$ (1), Galeazza (Pepoli-Crevalcore), $15 \mathrm{~m}$ (2), dintorni di Cento, $15 \mathrm{~m}$ (3). La specie è stata segnalata anche di altre località, dove però non ci è stato possibile confermarla.

collina che in montagna. Per Bonizzi (1870) la specie era rappresentata nel Reale Museo di Modena da "diversi esemplari presi sulle montagne modenesi. Io stesso ne osservai e ne raccolti diversi individui nel comune di Zocca" ". Secondo de Marchi (1980), che ha attinto anche da Del Prato (1887, 1899), nel Parmense il serpente preferisce le "plaghe più aride e sassose del colle e del monte: è però frequente anche nella pianura bassa". Per Camerano (1891) nella "valle del Po non è rara".

Dall'ampia letteratura sulla distribuzione e sulla biologia di questa specie -elemento faunistico arboreale mediterraneo-caspico, a corologia euroanatolico-caucasica con riduzione delle porzioni estreme SW, SE e settentrionali dell'areale - risulta che il rettile sia un'animale a larga valenza eco- 
logica, e cioè eurizonale o xenoalpino perché nella stessa area geografica, per la sua euriecia o capacità di vivere in condizioni ambientali molto variabili, può estendersi dal piano basale o da quello collinare fino al piano montano o al piano culminale. Nel Bolognese (fig. 14), tuttavia, non ci sono noti, per il momento, colubri lisci sorpresi oltre i $700 \mathrm{~m}$ d'altitudine; e i pochi esemplari trovati (la maggior parte di quelli raccolti durante la "Campagna Antivipera" sono di provenienza ignota) sono stati osservati ai margini di coltivi e di pascoli con frequenti siepi e modeste estesioni di basse e miste boscaglie caducifoglie.

\section{Biacco [Coluber viridiflavus (Lacepède, 1789)]}

In Emilia-Romagna, Zaffagnini e Bigazzi (1975) scrivono che "la colorazione del dorso", di questo veloce e mordace colubro, "varia da verde scuro a nero con fitte macchie gialle", mentre "quella del ventre è bianco sporco; inoltre "non è difficile trovare esemplari melanici".

Nel Bolognese (fig. 15) la specie sfoggia soprattutto due livree, le cui caratteristiche ornamentali e cromatiche sembrano passare più o meno gradualmente da una livrea all'altra in base all'ambiente ecologico e quindi ai tipo di vegetazione o alle zone bioclimatiche.

In linea generale sembra possibile ipotizzare che nelle aree climatiche di transizione della Padania -e cioè nel climax del farneto-carpineto del piano basale (0-100 $\mathrm{m}$ circa d'altitudine) e nei settori inferiore e mediano del climax della roverella del piano collinare (100-400/450 m circa d'altitudine)- siano più frequenti i biacchi a dorso grigio-nerastro, con numerose $\mathrm{e}$ piccole vermicolature, di solito biancastre, disposte in genere in senso longitudinale (tav. 8); mentre, nel settore superiore del climax del roverelletocerreto del piano collinare $(400 / 450-600 / 700 \mathrm{~m}$ circa d'altitudine) e nel climax del faggio (600/700-1.500/1.600 m circa d'altitudine) sembrano prevalere, almeno fino a circa $1.300 \mathrm{~m}$ di quota, $\mathrm{i}$ biacchi a dorso nero indaco, con vermicolature gialle disposte di regola in senso trasversale. In entrambi i casi, il ventre è giallo o giallo-biancastro punteggiato di grigio e di nero soprattutto ai lati delle piastre, dove in alcuni casi sapiccano macchie circolareggianti blu o celesti (tav. 9).

Le forme melaniche e melanotiche sembra che siano più comuni tra le popolazioni della Romagna (Silvestri 1972, Senni 1985).

Non abbiamo mai trovato individui albinotici, segnalati nel Modenese da Carruccio (1882a e recentemente confermati ${ }^{12}$ sulla base di un maschio adulto catturato presso Nonantola nel 1978.

La validità tassonomica e sistematica delle sottospecie (per esempio antoniimanueli, carbonarius, kratzeri, viridiflavus) del polimorfo biacco è stata sostenuta da tanti studiosi più o meno tradizionalisti. A questa lunga fase è seguita quella - molto più breve, potenziale e sperimentale- di coloro che vedevano nelle due principali livree del rettile (la verde-gialla e la nera) non due sottospecie, ma o due supposte specie o due varianti individuali 


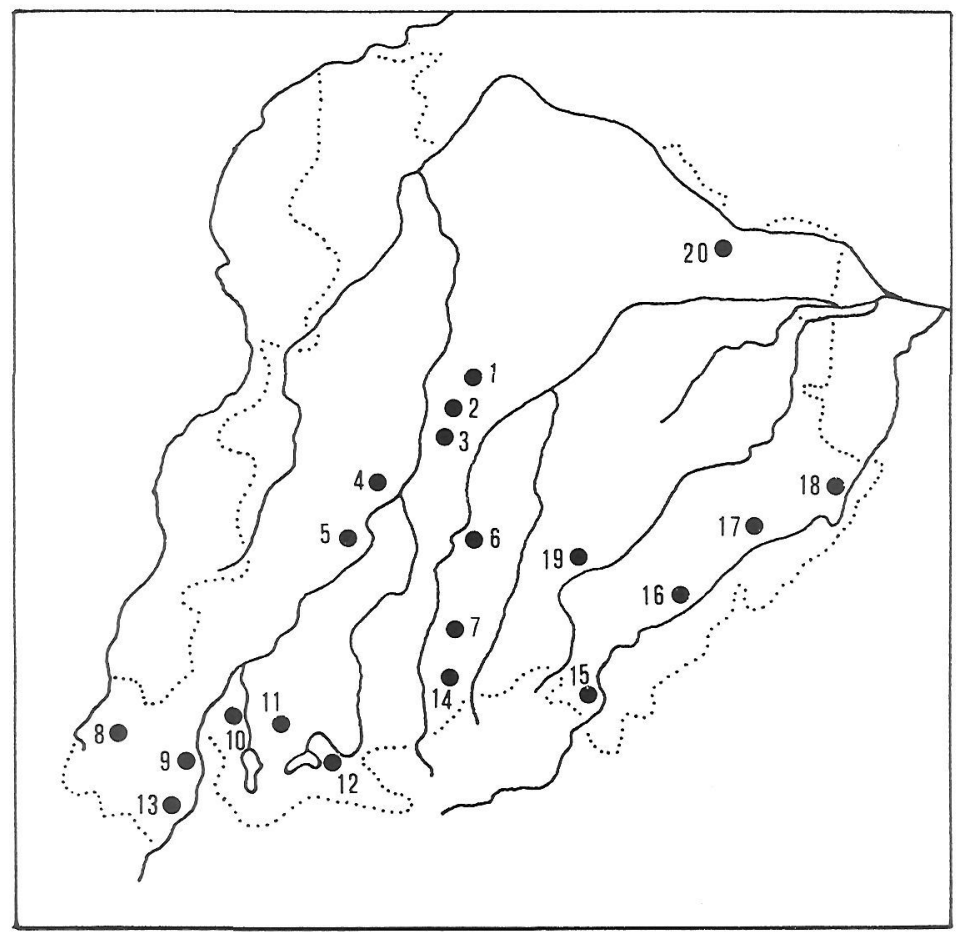

Figura 15.- Distribuzione del biacco (Coluber viridiflavus) nella provincia di Bologna.

Bentivoglio, $19 \mathrm{~m}$ (1), Bologna, $52 \mathrm{~m}$ (2), Borgo Panigale-Casaglia-Farneto, 52-230 m (3), Monte Mario e Sasso Marconi, 128-400 m (4), Marzabotto (5), Pianoro e Val di Zena, 200-550 m (6), Loiano e Roncastaldo, 600-714 m (7), Lizzano in Belvedere, $640 \mathrm{~m}$ (8), Porreta Terme, L'Olmo, Madonna del Faggio, 349-825 m (9), Casola, 620 m (10), Camugnano, 692 m (11), Castiglione dei Pepoli e dintorni, 691-1.100 m (12), da Granaglione al Rifugio del Monte Cavallo, 780-1.280 m (13), Monghidoro, $841 \mathrm{~m}$ (14), Castel del Rio, $200 \mathrm{~m}$ (15), Casalfiumanese, $125 \mathrm{~m}$ (16), Imola e dintorni 47-100 m (17), Mordano, $21 \mathrm{~m}$ (18), Monterenzio e dintorni, Bisano-Villa Sassone, San Clemente, 235-800 m (19), Molinella, 8 m (20)

(esemplari con l'una o l'altra delle livree coabitano in più località dell'areale). Oggi, l'ipotesi delle due specie, dopo le ricerche svolte da vari erpetologi, è stata abbandonata e ha trovato più sostegno quella di considerare il biacco specie monotipica ${ }^{13}$. 


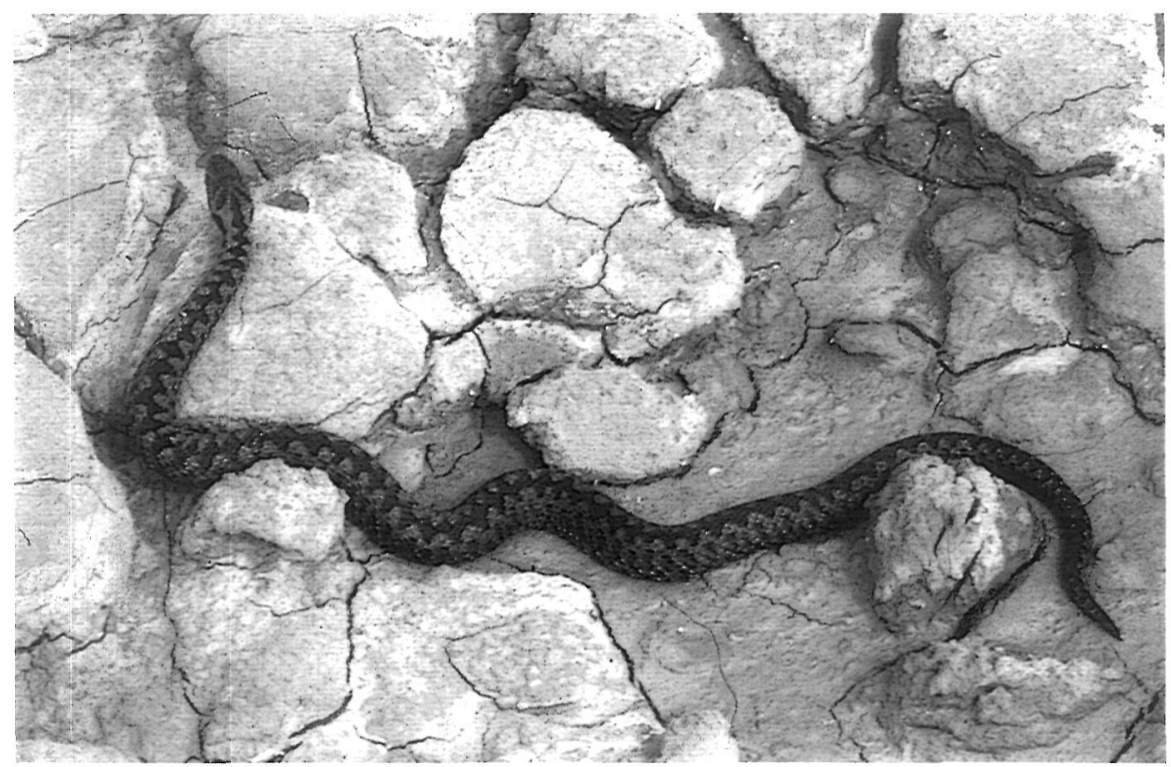

Tav. 1. - Marasso (Vipera berus), lungo all'incirca $38 \mathrm{~cm}$, sorpreso in località Salse di Nirano (Modena) a circa $200 \mathrm{~m}$ d'altitudine, nell'estate 1982 (foto Luciano Callegari).

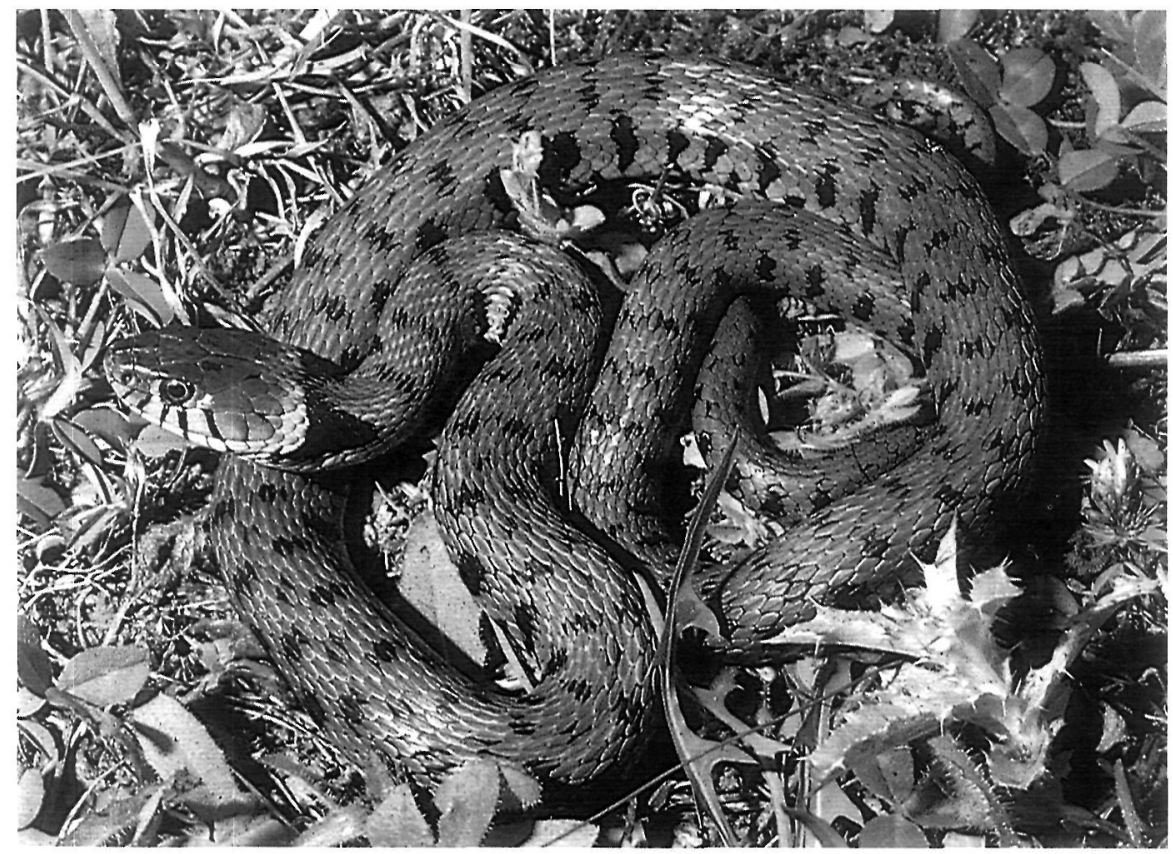

Tav. 2.- Femmina adulta di biscia dal collare (Natrix natrix) della varietà lanzai (foto Paolo Mazzei). 


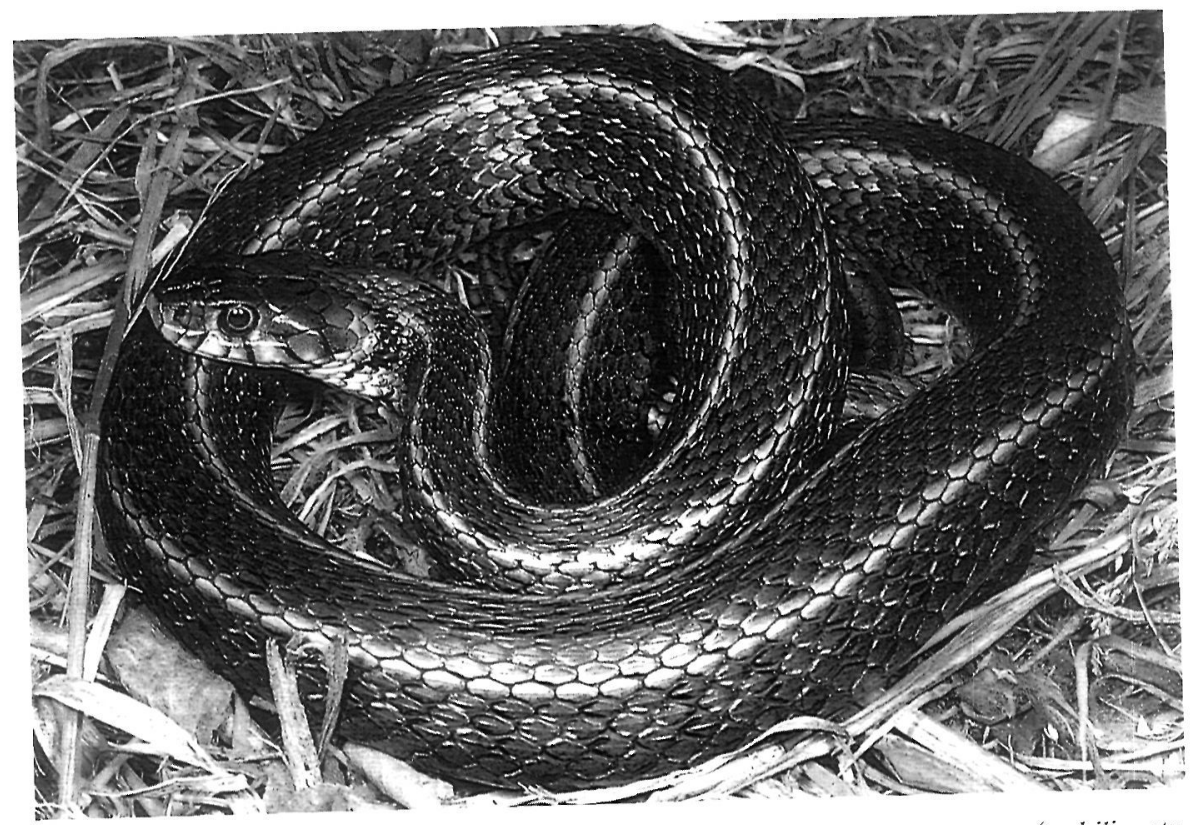

Tavia dal collare (Natrix natrix) della varietà persa (= bilincata,

Tav. 3. - Femmina adulta di biscia dal collare (Natrix natrix) della
dalmatina, lineata) lunga $130 \mathrm{~cm}$ (foto Paolo Mazzei).

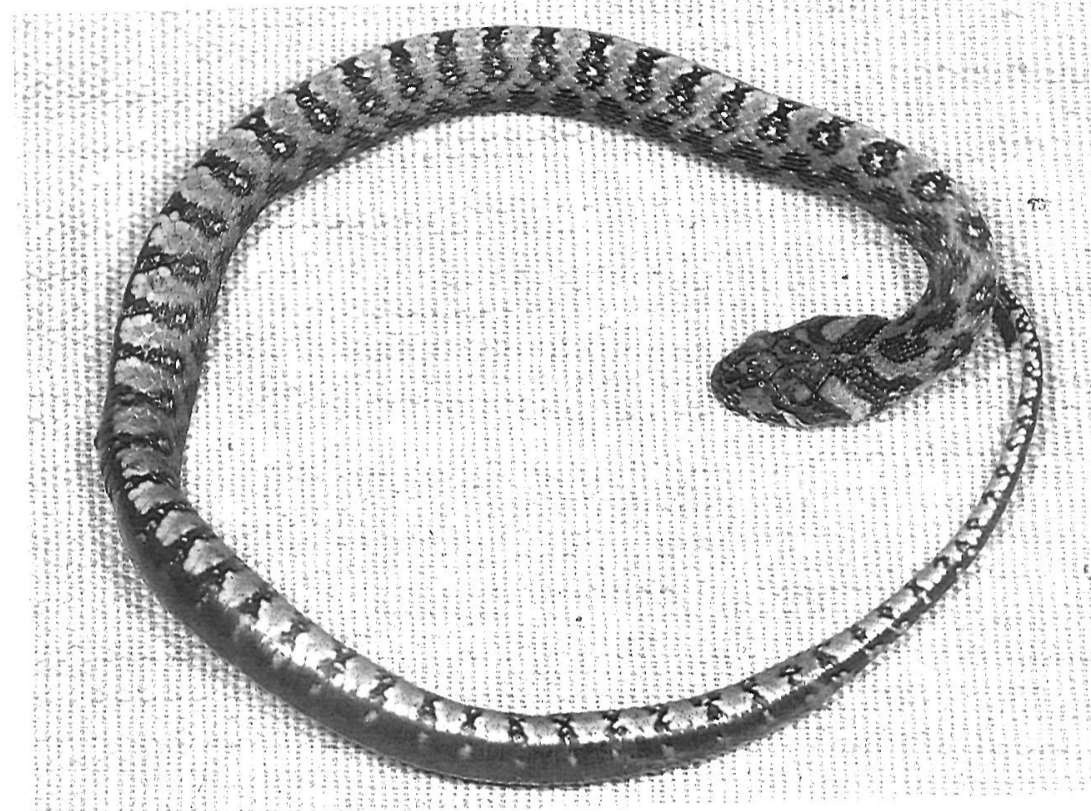

Tav. 4.- Giovane biscia viperina (Natrix maura) dei dintorni di San Secondo Parmense (foto Paolo Mazzei). 


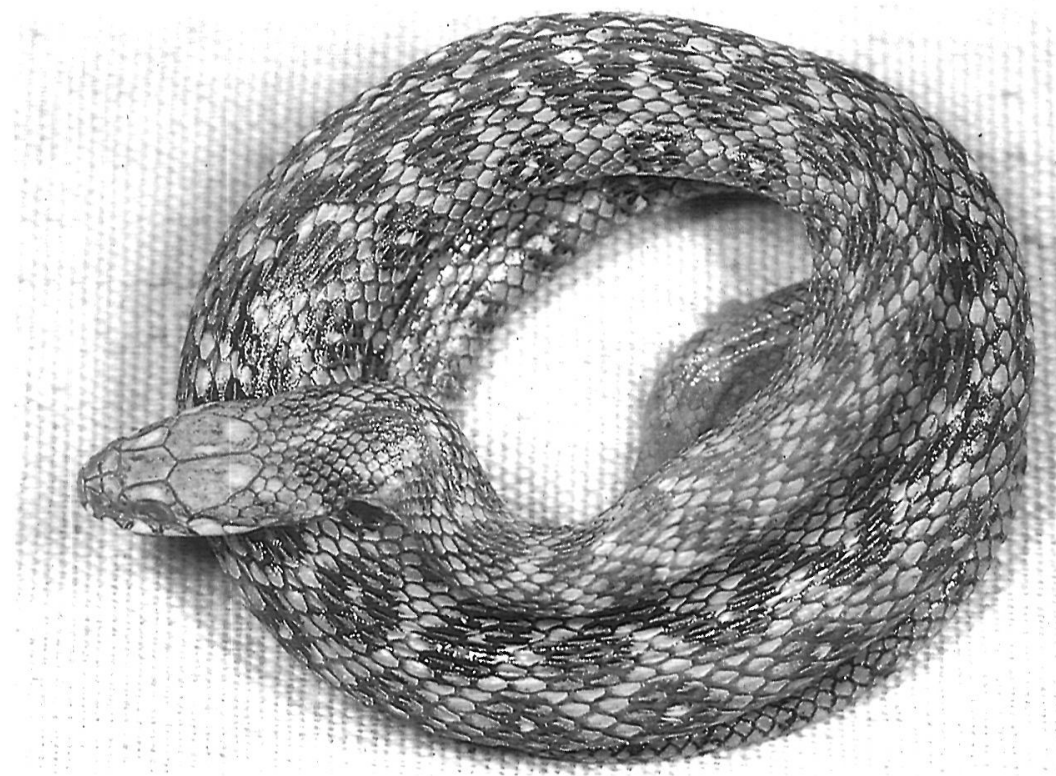

Tav. 5.- Giovane biscia viperina (Natrix maura) dei dintorni di Santa Maria di Zena: località Fazzola (foto Paolo Mazzei).

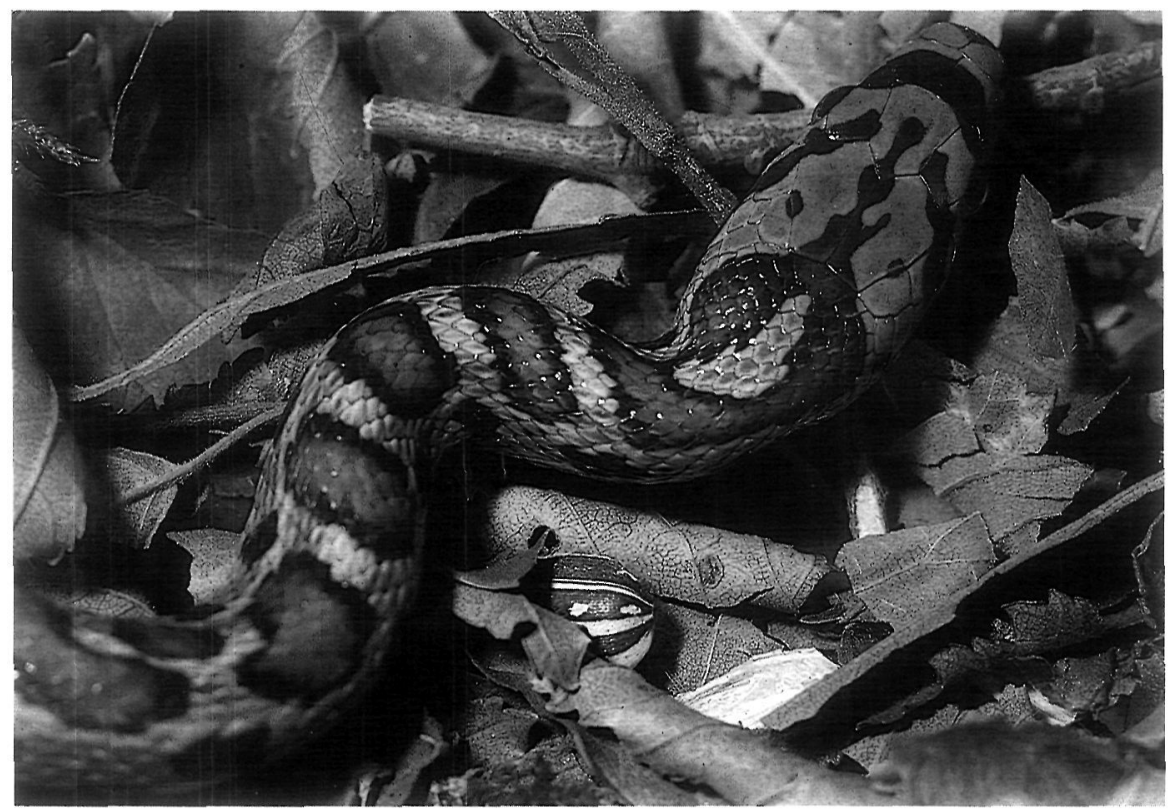

Tav. 6.- Primo piano di un maschio adulto di colubro leopardino (Elaphe situla) della varietà leopardina (foto Giovanni Papini). 


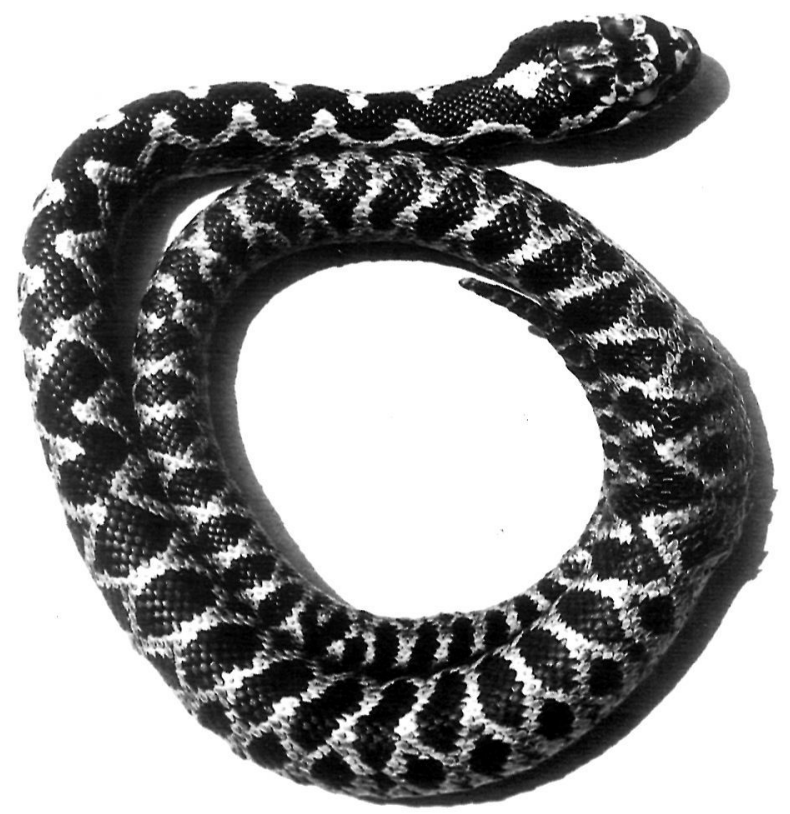

Tav. 7.- Livrea di un cervone (Elaphe quatuorlineata) giovane (foto Silyio Bruno).

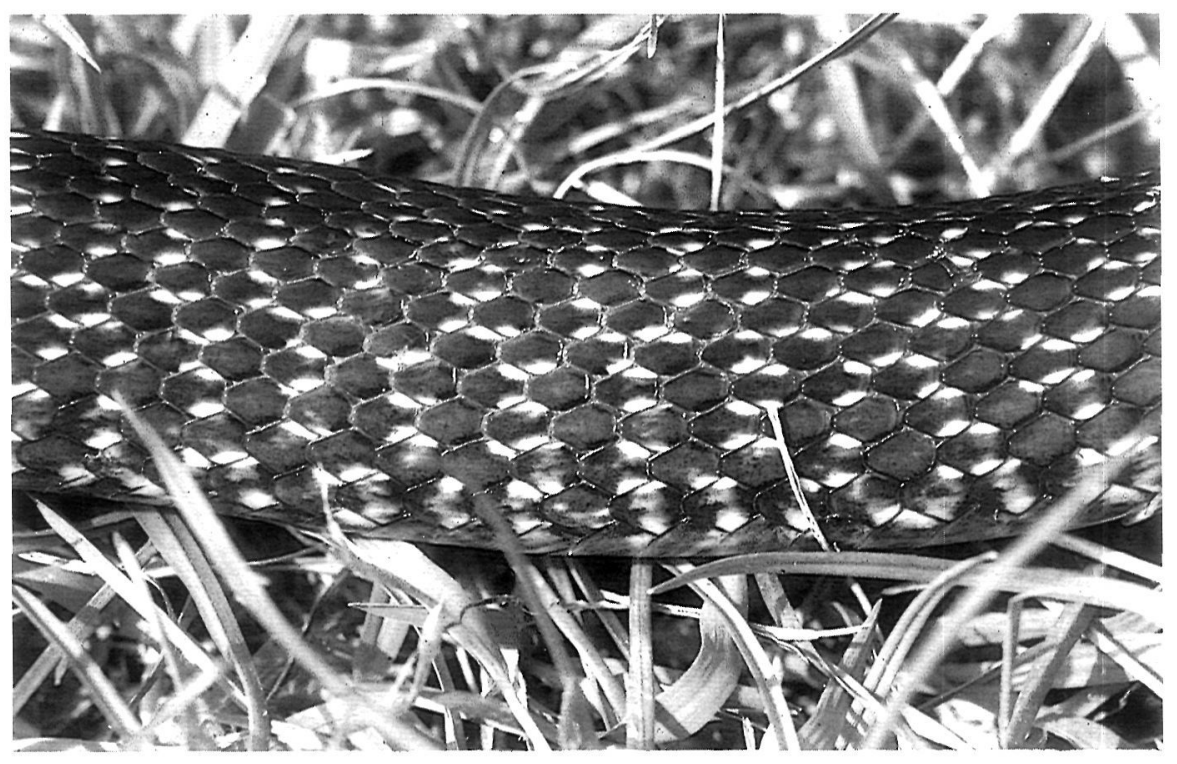

Tav. 8.- Dorso di un biacco (Coluber viridiflarus) adulto di Villa Ghigi (Bologna). Questa livrea sembra che sia dominante nei territori comunali del settore occidentale del Bolognese e nei biacchi che vivono in località del piano basale nel climax del querceto-carpineto (foto Guglielmo Stagni). 


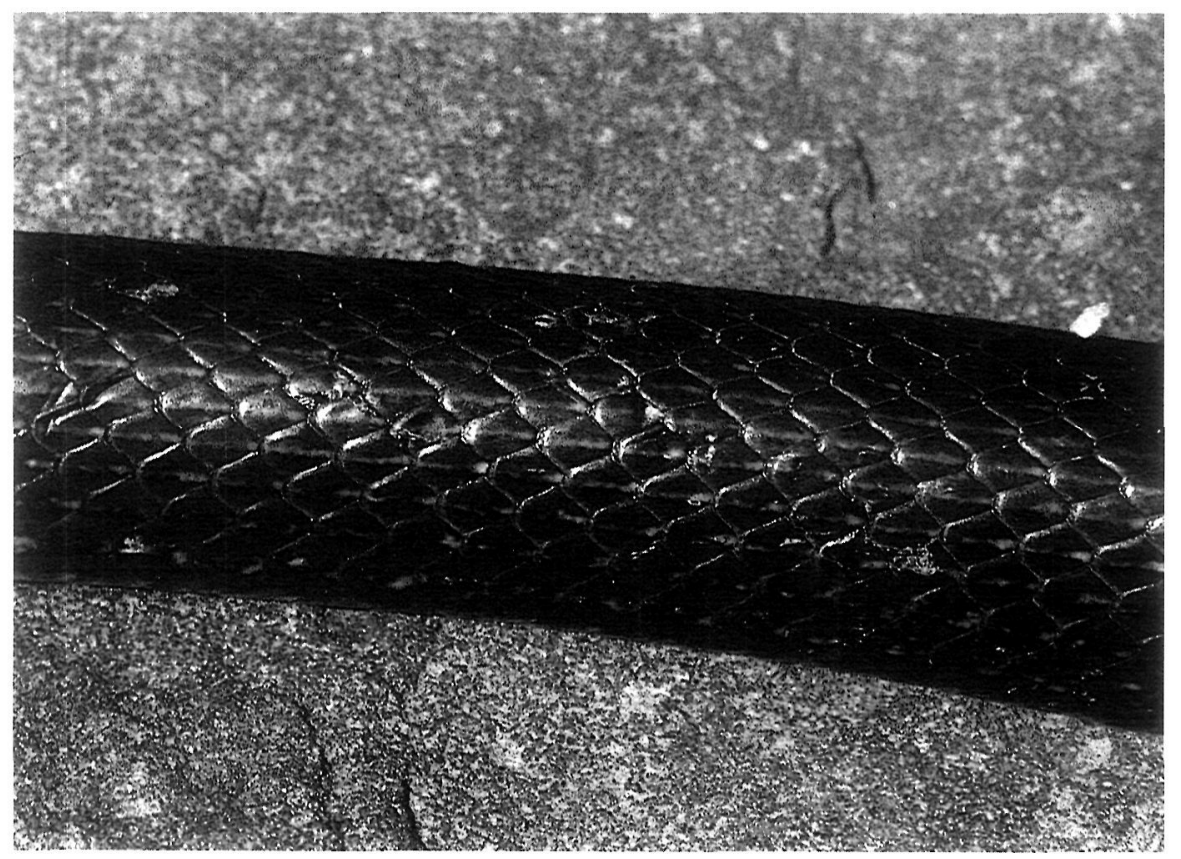

Tav. 9.- Dorso di un biacco (Coluber viridiflavus) adulto di la Martina ( $860 \mathrm{~m}$ circa di altitudine, alta valle dell'Idice, Loiano). Questa livrea — che in provincia di Bologna sostituisce quella nera caratteristica, in Emilia, della Romagna - sembra che sia predominante nei territori comunali del settore orientale del Bolognese e nei biaachi che vivono in località collinari e submontane (dai 450 ai circa $1.300 \mathrm{~m}$ di quota) nei climax della roverella-rovere, del faggio e dell'abete bianco (foto Guglielmo Stagni). 


\section{RINGRAZIAMENTI}

Ringraziamo molto sentitamente Angelo Ciucci (Porretta Terme), Augusta Bellei (Fiorano Modenese), Paolo Boldreghini (Bologna), Luciano Callegari (Fiorano Modenese), Giuseppe Cherpelli (IBACN, Bologna), Adolfo Fiori (Roma), Corradino Guacci (Campobasso), Aldo Imperiale (Modena), Sergio Mezzadri (San Nicolò, Piacenza), Manuel Mongini (Parma), Lucia Naviglio (ENEA, Casaccia, Roma), Mario Spagnesi (INFS, Bologna), Stefano Zilocchi (Piacenza) c Marco Zuffi (olim Milano, nunc Pisa) per la loro squisita collaborazione. Un espressione di gratitudine anche a tutti i "serpari" bolognesi (agricoltori, cacciatori, contadini, escursionisti, forestali, ecc.) senza il cui fattivo contributo questa nota non avrebbe visto la luce.

\section{NOTE}

${ }^{1}$ La più massiccia, tra le "campagne antivipera" promosse in Italia dall'ultimo dopo guerra a oggi, sembra che sia stata quella patrocinata dalla "Sezione Provinciale Cacciatori" di Vicenza tra il $1951 \mathrm{e}$ il 1961. In 11 anni furono uccise nel vicentino 18.953 vipere o presunte tali. I premi erogati erano di "200 lire, per ogni vipera consegnata al guardiacaccia della zona o direttamente al presidente provinciale dott. Ennio Fabrello nei mesi di maggio e giugno, e di 100 lire nei mesi successivi" (cfr. i vol. 51-55, 1956-1960), della rivista venatoria fiorentina Diana).

2 Per maggiori chiarimenti rimandiamo alla storia della specie nella Pianura Padana tentata da S. Bruno (Soc. vencz. Sci, nat., Venezia, vol. 5, suppl., pp. 1-70, 1980).

${ }^{3}$ Sull'argomento rimandiamo, per esempio, agli scritti di A. P. Ninni (Atti Soc. ital. Sci. nat., Milano, vol. 22, pp. 175-181, 1879 e vol. 23, pp. 171-186, 1880) e a quello di E. De Betta (Atti r. Ist. veneto Sci. Lett. Art., Venezia, ser. 5, vol. 6, pp. 359-392, 1880).

${ }^{4}$ Se consiceriamo la più tipica livrea italiana di Vipera berus e quella analoga di Vipera aspis in provincia di Modena o in tutta l'Emilia-Romagna, è più verosimile che l'A. si riferisse all'ornamentazione piuttosto che alla colorazione.

"Con il nome di "salse" si indicano emissioni di idrocarburi che, attraverso fratture, escono alla superficie trascinando acqua salata e fango freddo, che costituiscono alla sommità apparati a forma di cono dai quali gorgogliano i gas infiammabili. Nel Preappennino Emiliano le salse sono attualmente presenti nelle provincie di Bologna, Modena, Parma e Reggio Emilia. Per informazioni sull'argomento rimandiamo a: C. Ferrari et alii (1985). Le salse dell'EmiliaRomagna. Guida escursionistica. Assessorato all'Ambiente e alla Difesa del suolo della Regione Emilia-Romagna, Bologna, $152 \mathrm{pp}$.

6 Il testo relativo al marasso fu pubblicato, con più illustrazioni e meno testo dell'attuale, suila rivista parmense Ambiente e Natura del Po e degli Appennini (vol. 4, fasc. 1, pp. 25-28, 1988)

${ }^{7}$ Sull'argomento rimandiamo, per esempio, ai contributi di E. Calabresi (Boll. Ist. zool. $r$. Univ. Roma, vol. 2, pp. 78-127, 4 tav. ft, 1924), di L. Capocaccia (Ann. Mus. civ. Stor. nat., Genova, vol. 70, pp. 173-192, 2 tav. ft, 1958) e di S. Bruno (Atti Soc. ital. Sci. nat.. Milano, vol. 117, pp. 165-194, 1 tav. ft, 1976).

8 A questo proposito ricordiamo che ancora negli anni Sessanta dell'attuale secolo, alcuni appassionati naturalisti determinarono, come colubri leopardini, giovani cervoni ostentati dai serpari a Cucullo (Abruzzo), durante la festa di San Domenico (il primo giovedi di maggio) e che queste affermazioni furono riprese da più quotidiani locali e nazionali, oltre che dalla radio.

${ }^{9}$ La presenza e la distribuzione della specie in Calabria e nella Basilicata ionica devono cssere approfondite. Mentre in Basilicata orientale (provincia di Matera) la presenza del colubro leopardino è stata attestata (negli anni Ottanta), le notizie più recenti per la Basilicata SE e la Calabria risalgono perlopiù agli anni Cinquanta e Sessanta del XX secolo c, sebbene siano state poi riprese da più Aa., non ci risulta che siano state confermate.

${ }^{10}$ Secondo la disgnosi formulata da L. Capocaccia (Ann. Mus. civ. Stor. nat., Genova, vol. 74, pp. 353-387, 1964).

11 Gli esemplari cmiliani ricordati da S. Bruno (Atti Accad. Groenia Sci. nat. Catania, ser. 6. vol. 18, p. 109, 1966) provenivano tutti dal Modenese.

${ }^{12}$ Cfr. S. Vanni e B. Lanza (Natura, Milano, vol. 70, fasc. 1-2, pp. 94-96, 1979).

13 Cfr. per esempio B. Shätti e S. Vanni (Revue suisse Zool., Genève, vol. 93, fase. 1, pp. 219-232, 1986). 


\section{BIBLIOGRAFIA}

ALDROVANDI, U.; AMBROSINO, B. 1639. Serpentum et Draconum Historiae, C. Ferronij, Bononiae, $478 \mathrm{pp}$.

AUTORI VARI, 1986. Il fiume vivente. Ecologia e aspetti naturalistici del fiume Panaro. $P_{i-}$ cus (Suppl.), Modena, 11 (3): 1-128.

BAGNARESI, U.; FERRARI, C., coord., 1987. I boschi dell'Emilia-Romagna, Assess. Ambiente e dif. suolo Reg. Emilia-Romagna, Bologna, $268 \mathrm{pp}$

BASSI, S., 1989. Un ritrovamento erpetologico insolito per l'Emilia-Romagna. Natura e Montagna, Bologna, 36 (3-4): 36.

[BELLEI, A.; PARISI, A.; TERMANINI, R.; CALLEGARI, L.], 1984. BE.PA.TE.CA. è un gruppo di studiosi dell'ambiente naturale. Comune di Fiorano, 8 (6): 5.

BERNARDINO, A.; ASCHIERI, B.; FERRARIS, T.; PANERO, R., 1977. La fauna dalla Pianura Padana alle Alpi. Gribaudo, Cavallermaggiore (Cuneo), $266 \mathrm{pp}$.

BERTOLANI MARCHETTI, D., 1980. Fiorano e il suo territorio. L. Parma S.p.A., Bologna, 24 pp.

BERTOLANI MARCHETTI, D., 1982. Vicende climatiche passate e attuali alla luce di recenti ricerche. Atti $10^{\circ}$ Conv. Meteorologia appenninica, Reggio Emilia, pp. 613-625.

BERTOLANI MARCHETTI, D., 1983. Fiorano e la sua vegetazione. ARBE S.p.A., 36 pp.

BERTUSI, M.G., 1982. La fauna della Provincia di Reggio Emilia (pp. 60-79). In: Aa.Vv. Guida alla natura del Reggiano. Amministr. prov. Reggio Emilia e Assessor. pianif. territor., tutela ambient e difesa del suolo, Reggio Emilia, $80 \mathrm{pp}$.

BIANCONI, G., 1867. indicazioni sulla zoologia di Porretta (pp. 181-192). In: Aa.Vv. Illustrazioni delle Terme di Porretta e del suo territorio. Tip. Regia, Bologna, $472 \mathrm{pp}$.

BOLDREGHINI, P., 1983. La fauna: i Vertebrati. In: PICCOLI, F., coord. Aspetti naturalistici di alcune zone umide di acqua dolce della bassa Pianura Padana. Assess. Ambiente c dif. suolo Reg. Emilia-Romagna, Bologna, pp. 127-162.

BOLDREGHINI, P., 1987. La fauna: i Vertebrati. In: BAGNARESI, U.; FERRARI, C., coord. I boschi dell'Emilia-Romagna. Assess. Ambiente e edif. suolo Reg. Emilia-Romagna, Bologna, pp. 135-163.

BONIZZI, P., 1870. Enumerazione sistematica dei Rettili e Anfibi finora raccolti e studiati nel Modenese. Eco dell'Univ., Modena, 1 (18-22): 1-24 (estratto).

BONIZZI, P., 1881. Primo catalogo della collezione dei prodotti naturali della Provincia Modenese finora raccolti, studiati e classificati nel gabinetto di storia naturale dell'Istituto Tecnico Provinciale a spese de Prof. P.B. [Paolo Bonizzi]. Tip. P. Toschi \& C., Modena, $186 \mathrm{pp}$.

BOULENGER, G. A., 1894. Catalogue of the snakes in the British Museum (Natural History). Taylor and Francis, London, 2: XII-382, 20 tavv. ft.

BOULENGER, G. A., 1896. Catalogue of the snakes in the British Museum (Natural History). Taylor and Fracis, London, 3: XIV-728, 25 tavv. ft.

BRUNO, S., 1992. Repertorio zoogeografico, geonemico, tassonomico, biografico e bibliografico degli studiosi e degli studi di erpetologia italiana. I. Serpentes: 1800-1899. Atti Accad. rover. Agiati, (7) 1 (B): 1-256, 1991.

BRUNO, S.; MAUGERI, S., 1977. Rettili d'Italia. II. Serpenti. A. Martello-Giunti, Firenze, 208 pp. [Nell'edizione del 1985, a pag. 22, fig. 8, invece di "colubro di Ricciolii" leggi "biscia dal collare"].

BRUNO, S.; MAUGERI, S., 1990. Serpenti d'Italia e d'Europa. G. Mondadori, Milano, 224 pp.

CAMERANO, L., 1889. Monografia degli Ofidi italiani. Parte prima. Viperidi. Mem. R. Accad. Sci. fis. mat. Torino, (2) 39: 195-243.

CAMERANO, L., 1891. Monografia degli Ofidi italiani. Parte seconda: Colubridi, e monografia dei Cheloni italiani. Mem. R. Accad. Sci. fis. mat. Torino, (2) 41: 403-481.

CARLONI, G. C.; FRANCAVILLA, F., 1974. L'ambiente fisico. In: CANTELLI, F.; GUGLIELMI, G.; MASSERA, M., coord. L'Emilia-Romagna. Teti Ed., Milano, pp. 9-21.

CARRUCCIO, A., 1882a. [Sull'albinismo nell'uomo e negli altri Vertebrati. Elenco degli animali albini, melanici ed isabellini della Collezione dei Vertebrati del Museo Zoologico dell'Università di Modenal. Annali Soc. Natural. Modena, (2) 15: 17-19.

CARRUCCIO, A., 1882b. Importanza ed utilità delle collezioni faunistiche locali e contribuzione alla fauna dell'Emilia (Vertebrati del Modenese). Annali Soc. Natural. Modena, (2) $15: 130-184$.

CIUCCI, P.A.; FUMAGALLI, D., 1981. La fauna (pp. 27-28). In: Una valle da scoprire. Valle del Randaragna dell'Alta Valle del Reno. Scuola Grafica Salesiana, Bologna, 277 pp. 
COCCONI, G., 1881. Cenni sulla fauna della parte elevata della provincia di Bologna (pp. 121-160). In: BOMBICCI, L. L'Appennino Bolognese. Descrizioni e itinerari. Tip. Fava e Garagnani, Bologna, $888 \mathrm{pp}$.

CORBETTA, F., 1974. La vegetazione. In: CANTELLI, F.; GUGLIELMI G.; MASSERA, M., coord. L'Emilia-Romagna. Teti Ed., Milano, pp. 22-33.

DE BETTA, E., 1868. I rettili ed anfibi della Grecia con alcune notizie sulla distribuzione geografica delle specie. Atti R. Ist. ven. Sci. Lett. Art., Venezia, (3) 13 [1867-1868] (2): 876-963.

DE BETTA, E., 1874. Fauna d'Italia. Anfibi e Rettili. F. Vallardi, Milano, 107 pp.

DE BETTA, E., 1879. Nuova serie di note erpetologiche per servire allo studio dei rettili ed anfibi d'Italia. Atti $R$, ist. ven. Sci. Lett. Art., Venezia, (5) 5: 378-415.

DE BETTA, E., 1880a. Sulla distribuzione geografica dei serpenti velenosi in Europa e più particolarmente nell'Italia. Atti $R$. Ist. ven. Sci. Lett. Art., Venezia, (5) 6: 359-392.

de MARCHI, A., 1980. Nuova Guida Naturalistica del Parmense. E. Albertelli Ed., Parma, 182 pp. [La 1." ediz., pubblicata da "La Nazionale", è del 1974].

DEL PRATO, A., 1887. Rettili e Anfibi della Provincia di Parma. Boll. Comit. agrar, parmense, Parma, (7-8): 1-7 (estratto).

DEL PRATO, A., 1899. I Vertebrati della Provincia di Parma. Tip. L. Battei, Parma, VIII-82 pp.

DUMÉRIL, A.M.C. e BIBRON, G., 1854. Erpétologie Générale ou Histoire Naturelle Complète des Reptiles. Libr. Encyclop. De Roret, Paris, 7 (1): 1-780, (2): 781-1.536.

FERRARI, C. et alii, 1980. Flora e vegetazione dell'Emilia-Romagna. Assess. Ambiente e dif. suolo Reg. Emilia-Romagna, Bologna, $340 \mathrm{pp}$.

GÜNTHER, A., 1858. Catalogue of Colubrine Snakes in the Collection of the British Museum. E. Newnam, London, XVI-281 pp.

IMPARATI, E., 1940. I Rettili del Piacentino. Strenna, Piacenza, 18: 151-162.

JAN, G., 1863. Elenco sistematico degli Ofidi descritti e disegnati per l'Iconografia Generale. A. Lombardi, Milano, VII-143 pp.

LANZA, B., 1983. Guide per il riconoscimento delle specie animali delle acque interne italiane. Anfibi, Rettili (Amphibia, Reptilia). C.N.R. Prom. Qual. Amb. AO/1/205, Verona, 27: 1-196.

LATTIN, G. de, 1967. Grundriss der zoogeographie. G. Fischer, Jena, 602 pp.

LORENZINI, D., 1914. Fauna di Porretta (cap. XII-XV, pp. 154-220). In: Guida dei Bagni di Porreta e dintorni. N. Zanichelli Ed., Bologna, 394 pp.

LUGARO, G., 1957. Elenco sistematico dei rettili italiani conservati nella collezione di studio esistente presso il Museo di Storia Naturale di Milano, con brevi note critiche ed esplicative. Atti Soc. ital. Sci. nat., Milano, 96 (1-2): 20-36.

MËRTENS R., WERMUTH H., 1960. Die Amphibien und Reptilien Europas. W. Kramer, Frankfurt a. M., $264 \mathrm{pp}$.

MÜlLER, P., 1981. Arealsysteme und Biogeographie. E. Ulmer, Stuttgart, 704 pp.

NAULlEAU, G., 1966a. La biologie et le comportament prédateur de Vipera aspis au laboratoire et dans la nature. Bull. Bio. fr. belg., 99: 395-524.

NAULLEAU, G, 1966b. Etude complémentaire de l'activité de Vipera aspis dans la nature. Vie et Milieu, (C) 17 (1): 461-509.

NAVIGLIO, L.; NICOLAI, P.; RACALBUTO, S.; UCCELLI, R. e SALMOIRAGHI, G., 1989. Contributi per una valutazione preliminare dell'impatto sull'ambiente naturale del progettato bacino di Castrola. ENEA: COORBOL di Bologna \& PAS/SCAMB-ECOL del CRE Casaccia (Roma), 53 pp.

NIEKISCH, M., 1982. Zur Vorkommen der Vierstreifennatter (Elaphe quatuorlineata) in Nord-Italien. Salamandra, Frankfurt am Main, 18 (3-4): 352-354.

PICAGLIA, L., 1895. La Fauna (pp. 82-107). In: PANTANELLI, D. et alii. L'Appennino Modenese descritto e illustrato. Cappelli Ed., Rocca San casciano, XI-1.196 pp.

PICCOLI, F., coord., 1983. Aspetti naturalistici di alcune zone umide di acqua dolce della bassa Pianura Padana. Assess. Ambiente e dif. suolo Reg. Emilia-Romagna, Bologna, $196 \mathrm{pp}$.

PIGNATTI, S., 1979. I piani di vegetazione in Italia. Giorn. bot. ital., Firenze, 113: 411-428.

RABACCHI, R., 1984. Guida agli Uccelli e alla Natura della Provincia di Modena. LIPU di Modena \& Assess. Dif. Suolo Ambiente Riequil. Faun. Territ. Modena, Vignola, 176 pp.

ROSSI, G.; MEZZADRI, S., 1983. L'alta Val Nure nell'Appennino Piacentino. Natura e Montagna, Bologna, $30(2)$ : 49-60.

RUBBI, P.E., CLò O.T., 1980. L'Appennino Emiliano Romagnolo. Ente prov. Turismo BoIogna, Bologna, $128 \mathrm{pp}$. 
SAINT GIRONS, H., 1952a. Etude biogéographique des serpents paléarctiques. $C$. $R$. Soc. Biogéograph., 28 (250): 50-67.

SAINT GIRONS, H., 1952b. Ecologie et éthologie des Vipères de France. Ann. Sci. nat, Zool., Paris, (11) 14: 263-343.

SAINT GIRONS, H., 1971. Les vipères de l'Europe occidental. Rencontre, Lausanne, 33 pp.

SAINT GIRONS, H., 1975. Observation préliminaires sur la thermorégulation des vipères d'Europe. Vie et Milieu, (C) 25 (1): 137-168.

SAINT GIRONS, H., 1978. Thermorégulation comparée des Vipères d'Europe. Etude biotélemétrique. Terre et Vie, 32: 417-440.

SAINT GIRONS, H., 1982a. Influence des climats de type méditerranéen sur l'ecophysiologie et la répartition des Reptiles. Ecol. mediter., Marseille, 8 (1-2): 245-252.

SAINT GIRONS, H., 1982b. Remarques générales sur la biogéographie des reptiles méditeranèens. $P$. Cent. pir. Biol. exp., 13: 111-115.

SASSI, A., 1846. Rettili (pp. 149-150). In: ALBINI, L.; GANDOLFI Cr.; PALLAVICINO, M. C.; PARETO, L.; SPINOLA, M. Descrizione di Genova e del Genovesato. Ferrando, Genova, 1 (2): 1-180.

SCHÄTTI, B., 1982. Bermerkungen zur Okologie, Verbreitung und intraspezifischen Variation der Vipernatter, Natrix maura (Linné, 1758) (Reptilia, Serpentes). Rev. suisse Zool., Genève, 89 (2): 521-542.

SENNI, L., 1985. Gli anfibi e i rettili della Romagna (con particolare riferimento alla costa). Confed. N. Univ. Popol. Ital, Ordine Casa Matha, Ravenna, II-43 pp.

SILVESTRI, A., 1972. Panoramica sui Rettili ed Anfibi della Romagna (pp. 263-268). In: Osservazioni di Zoologia Romagnola. Tip. Moderna F.lli Zauli, Castrocaro Terme, 296 pp.

SILVESTRI, A., 1978. La vipera (Vipera aspis) in Romagna. Nota divulgativa sui Rettili e Anfibi della Romagna, con particolare riferimento alla vipera comune. Consigli pratici per difendersi dalle vipere. Tip. Moderna F.lli Zauli, Castrocaro Terme, $22 \mathrm{pp}$.

STAGNI, G., 1982. La fauna: Anfibi e Rettili (pp. 142-144). In: Aa.Vv. La collina di Bologna. Assessor. Program. Territ. Comune di Bologna, Bologna, $224 \mathrm{pp}$.

STAGNI, G., 1984. Osservazioni sulla distribuzione e sulla morfologia di Vipera aspis (Linnaeus, 1758) nel territorio della Provincia di Bologna. Univ. Bologna, Fac. Sci. mat. fis. nat., Corso Laur. Sci. nat. 1983-1984, 273 pp. [Di prossima pubblicazione].

STAGNI, G., 1986. Rettili (pp. 61-67). In: BERTUSI, M. G. Studio della fauna appenninica. Informazioni IBC, Bologna, NS, 2 (5): 55-72.

THORPE, R. S., 1975a. Biometric Analysis of Incipient Speciation in the Ringed Snake, $\mathrm{Na}$ trix natrix (L.). Experientia, Basel, 31: 180-181.

THORPE, R. S., 1975b. Quantitative handling of characters useful in snake systematics with particular reference to intraspecific variation in the Ringed Snake Natrix natrix (L.) Biol. Jour. Linn. Soc., London, 7 (1): 27-43.

THORPE, R. S., 1979. Multivariate analysis of the population systematics of the Ringed Snake Natrix natrix (L.) Proc. R. Soc. Edinburg, (B) 78: 1-62.

THORPE, R. S., 1980. Microevolution and taxonomy of European reptiles with particular reference ot the grass snake Natrix natrix and the wall lizards Podarcis sicula and $P$. melisellensis. Biol. Jour. Linn. Soc., London, 14: 215-233.

TOMASELLI, R. et alii, 1973. Carta bioclimatica d'italia. La vegetazione forestale d'Italia. Collana verde, Roma, 33: 1-64, 3 carte a col. ft.

TOSCHI, A., 1963. Fauna dell'Appennino tosco-emiliano (pp. 127-132). In: BORTOLOTTI, G. Guida dell'alto Appennino Bolognese-Modenese-Pistoiese, dalle Piastre all'Abetone. C.A.I. sez. Bologna-Modena-Montagna Pistoiese \& Tamarii Ed., Bologna, 678 pp.

TOSCHI, A., 1967. Vipere in aumento. Natura e Montagna, Bologna, 7 (1-2): 1-2.

TOSCHI, A., 1974. La fauna. In: CANTELLI, F., GUGLIELMI, G., MASSERA, M., coord. L'Emilia-Romagna. Teti Ed., Milano, pp. 34-40.

VANDONI, C., 1914. I rettili d'Italia. U. Hoepli, Milano, 274 pp.

ZAFFA GNINI, F. e BIGAZZI, M. 1975. I serpenti dell'Emilia-Romagna. Natura e Montagna, Bologna, 22 (3): 5-16.

ZANGHERI, P., 1961. La Provincia di Forlì nei suoi aspetti naturali. Tip. Moderna F.lli Zauli, Castrocaro Terme, XVI-390 pp.

ZANGHERI P., 1969. Repertorio sistematico e topografico della fauna vivente e fossile della Romagna. Mem. f.s. Mus. civ. Stor. nat. Verona, 1 (4): 1.415-1.963.

ZUFFI, M., 1983. Sulla presenza di Triturus alpestris apuanus e di Natrix maura nell'oltrepò pavese (Appennino Lombardo). Natura, Milano, 74 (1-2): 79-82. 